\title{
New Herbig Ae/Be stars confirmed via high-resolution optical spectroscopy $\star, \star \star$
}

\author{
A. Carmona ${ }^{1, \star \star \star}$, M. E. van den Ancker ${ }^{2}$, M. Audard ${ }^{1}$, Th. Henning ${ }^{3}$, J. Setiawan ${ }^{3}$, and J. Rodmann ${ }^{4}$ \\ 1 ISDC Data Centre for Astrophysics \& Geneva Observatory, University of Geneva, chemin d'Ecogia 16, 1290 Versoix, Switzerland \\ e-mail: Andres.Carmona@unige.ch \\ 2 European Southern Observatory, Karl Schwarzschild Str 2, 85748 Garching bei München, Germany \\ 3 Max-Planck Institute for Astronomy, Königstuhl 17, 69117 Heidelberg, Germany \\ ${ }^{4}$ ESA/ESTEC, Space Environments \& Effects (TEC-EES), 2200 AG Noordwijk, The Netherlands
}

Received 3 December 2009 / Accepted 20 March 2010

\begin{abstract}
We present FEROS high-resolution $(R \sim 45000)$ optical spectroscopy of 34 Herbig Ae/Be star candidates with previously unknown or poorly constrained spectral types. Within the sample, 16 sources are positionally coincident with nearby $(d<250 \mathrm{pc})$ star-forming regions (SFRs). All the candidates have reported infrared excess. We determine the spectral type and luminosity class of the sources, derive their radial and projected rotational velocities, and constrain their distances employing spectroscopic parallaxes and photometry from the literature. We confirm 13 sources as Herbig Ae/Be stars and find one classical T Tauri star. Three sources are emission line early-type giants (B, A, and F stars with luminosity class III) and may be Herbig Ae/Be stars. One source is a main-sequence Atype star. Fourteen sources are post-main-sequence giant and supergiant stars ( 7 with $\mathrm{H} \alpha$ emission and 7 without). Two sources are extreme emission-line stars and no accurate spectral classification was possible because of strong veiling. Most of the sources appear to be background stars at distances over $700 \mathrm{pc}$. We show that high-resolution optical spectroscopy is a crucial tool for distinguishing young stars (in particular Herbig Be stars) from post-main sequence stars in samples taken from emission-line star catalogs based on low-resolution spectroscopy. Within the sample, three young stars (CD-38 4380, Hen 3-1145, and HD 145718) and one early-type luminosity class III giant with emission lines (Hen 3-416) are at distances closer than $300 \mathrm{pc}$ and are positionally coincident with a nearby SFR. These 4 sources are likely to be nearby young stars and are interesting for follow-up observations at high-angular resolution. Furthermore, seven confirmed Herbig Ae/Be stars at $d>700$ pc (Hen 2-80, Hen 3-1121 N\&S, HD 313571, MWC 953, WRAY 15-1435, and Th 17-35) are inside or close $\left(<5^{\prime}\right)$ to regions with extended $8 \mu \mathrm{m}$ continuum emission and in their $20^{\prime}$ vicinity have astronomical sources characteristic of SFRs (e.g., HII regions, molecular clouds, dark nebulae, masers, young stellar-objects). These 7 sources are likely to be members of SFRs.
\end{abstract}

Key words. stars: pre-main sequence - stars: emission-line, $\mathrm{Be}$ - stars: fundamental parameters

\section{Introduction}

Herbig Ae/Be stars are intermediate-mass $\left(2-8 M_{\odot}\right)$ pre-main sequence (PMS) stars. They exhibit emission lines (e.g., $\mathrm{H} \alpha, \mathrm{H} \beta$, $\mathrm{Ca}$ II) in their optical spectra and infrared (IR) excess in their spectral energy distributions. These observational characteristics provide indirect evidence that Herbig Ae/Be stars have an accreting circumstellar disk. The infrared excess is interpreted as emission from small dust grains present in the hot surface layer of the disk. By analogy with the lower-mass T Tauri stars (e.g., Hartmann 1999; Muzerolle et al. 2004), the HI emission lines can be interpreted as originating in the magnetospheric

* Based on observations collected at the ESO-MPG $2.2 \mathrm{~m}$ telescope at la Silla Observatory, Chile (program IDs: 072.A-9006, 073.A-9008, 079.A-9014, 081.A-9003).

$\star \star$ Appendix is only available in electronic form at http://www. aanda.org

$\star \star \star$ This research was partly conducted while A. Carmona was at the Max-Planck Institute for Astronomy in Heidelberg and the European Southern Observatory in Garching. accretion shock ${ }^{1}$ when the gas of the disk reaches the surface of the star with free-fall velocities of a few hundred $\mathrm{km} \mathrm{s}^{-1}$. More recently, spatially resolved dust and molecular line observations in the millimeter and sub-millimeter domain (e.g., Mannings \& Sargent 1997; Semenov et al. 2005), together with scattered light coronographic imaging (e.g., Fukagawa et al. 2004, Grady et al. 2005) provided direct evidence that Herbig Ae/Be stars are effectively surrounded by a disk (for a detailed review about Herbig Ae/Be stars see Waters \& Waelkens 1998).

\footnotetext{
1 The origin of the $\mathrm{HI}$ emission lines in Herbig Ae/Be stars is in fact controversial. Several authors have alternative scenarios for magnetospheric accretion (e.g., strong stellar winds, outflows, direct disk accretion) to explain the origin of the H I lines (e.g., Böhm \& Catala 1993; Mottram et al. 2007). The magnetospheric accretion model can explain the $\mathrm{H} \alpha$ line and its spectropolarimetry signal in Herbig Ae stars (e.g., Pontefract et al. 2000; Vink et al. 2002, 2005). However, this is less clear in the case of Herbig Be stars, since wind or outflows contributions to the $\mathrm{H} \alpha$ line are likely, and the spectropolarimetry signal does not unambiguously support the magnetospheric accretion scenario (Mottram et al. 2007). As several stars in our sample are Herbig Be stars, it could well be that different emission mechanisms work for different stars in our sample.
} 
From the observational point of view, bright nearby $(d<250 \mathrm{pc})$ Herbig Ae/Be stars (and CTTS) are particularly relevant, because they permit detailed studies of the structure of their disks. Disks are interesting because they play a key role in early stellar evolution and are the sites of planet formation. In nearby sources, the disk can be spatially resolved with 8-10 m class telescopes and infrared and mm interferometers. In bright sources, high-resolution spectroscopy in the near and mid-IR can be obtained to study the gas in the disk (see reviews by Najita et al. 2007; and Carmona 2010). Since the amount of identified nearby PMS with spatially resolved disks is still relatively small, the identification of bright nearby Herbig Ae/Be stars is an important step for future observational studies of protoplanetary disks.

Herbig Ae/Be stars were initially identified based on the presence of emission lines (i.e. $\mathrm{H} \alpha$ ) in their optical spectra and their physical association with a dark cloud or nebulosity (e.g., Herbig 1960). Herbig (1960) used the last condition to exclude the post-main sequence $\mathrm{B}[\mathrm{e}]$ stars (i.e. giant or supergiant B-type stars with emission lines). Thanks to the advent of IR space observatories such as IRAS, ISO, and Spitzer this last criterion has been relaxed and replaced by the presence of near- or far-IR excess, in addition to the emission lines, as membership criteria to the Herbig Ae/Be stellar group (e.g., Finkenzeller \& Mundt 1984; Thé et al. 1994; Vieira et al. 2003).

However, published samples of Herbig Ae/Be stars may well be contaminated with other classes of objects. One should bear in mind three aspects of the identification: (i) in general, Herbig Ae/Be star candidates have been identified in surveys for emission-line stars based on low-resolution data, in particular slit-less spectra (this makes no difference on the detection of the emission lines, but it matters for the determination of the luminosity class, see below); (ii) post-main sequence $B[$ e] supergiants can also have IR excess (e.g., Miroshnichenko et al. 2005, and references therein); and (iii) as beam sizes for infrared observations employed in previous studies have typically been large (e.g., 30" in the case of IRAS), confusion with other infrared sources may have occurred.

Since hydrogen lines are observed in emission in Herbig Ae/Be stars, the hydrogen lines width, the usual means for determining the luminosity class, cannot be used. Thus the observation of gravity sensitive lines (e.g., N II at $3995 \AA$, Si II at 4128 and $4131 \AA, C$ II at $4267 \AA$, Si III at 4553 and $4561 \AA$, and $\mathrm{O}$ II lines at 4070 and $4976 \AA$ ) is required. However, these lines are relatively weak and are barely visible in low-resolution spectra. Therefore, low spectral resolution studies that have identified Herbig Ae/Be candidates have the important limitation that background $\mathrm{B}[\mathrm{e}]$ supergiants can be mistakenly classified as Herbig Ae/Be stars. Consequently, to confirm that a Herbig Ae/Be candidate is indeed a young star - and not a post-main sequence object - observations at high spectral resolution are necessary.

In this paper, we present the results of a high-resolution optical spectroscopy campaign aimed at identifying and characterizing new nearby Herbig Ae/Be stars. We obtained FEROS high-resolution $(R \sim 45000)$ optical spectra of 34 candidates to Herbig Ae/Be stars. We studied sources positionally coincident with nearby $(d<250 \mathrm{pc})$ star-forming regions (SFRs) and "isolated" sources. Our goal was to determine whether the candidates belong to the Herbig Ae/Be stellar group by searching emission lines in their spectra and by determining their spectral type and luminosity class. We then constrained their distances employing spectroscopic parallaxes and derived their radial and projected rotational velocities. In the case of sources positionally coincident with SFRs, we used the estimated distance to determine whether the sources are members of nearby SFRs. Finally, for the confirmed Herbig Ae/Be stars that are not members of nearby SFRs, we searched the Spitzer archive for $8 \mu \mathrm{m}$ imaging and the SIMBAD database to find evidence for extended near-infrared emission and astronomical objects characteristic of SFRs (e.g., HII regions, molecular clouds, dark nebulae, masers, young stellar-objects). Our aim was to find evidence of whether these distant Herbig Ae/Be stars might be members of distant SFRs.

\section{Observations}

\subsection{Target selection}

We selected the sources from the catalog of Thé et al. (1994). This catalog is divided in six tables: Table 1 presents the Herbig Ae/Be members and candidate members. Table 2, F-type stars potential candidates. Table 3 , extreme emission line objects. Table $4 \mathrm{a} / \mathrm{b}$, other early type emission line stars with IR excess from Allen \& Swings (1976) and Dong \& Hu (1991) respectively. Table 5, non-emission line early type shell stars and young stellar candidates. Table 6 , emission line stars rejected as Herbig Ae/Be candidates. We concentrated only on sources from Tables $1-4 \mathrm{a} / \mathrm{b}$ that are observable from the Southern Hemisphere. First, we selected the sources of unknown or poorly constrained spectral types that are positionally coincident with nearby $(d<250$ pc) SFRs. Second, we selected isolated Herbig Ae/Be stars candidates of unknown or poorly constrained spectral type that exhibited $V$ band magnitudes brighter than 14 (i.e. to obtain a good quality spectrum in a reasonable amount of exposure time). To establish the association of a Herbig $\mathrm{Ae} / \mathrm{Be}$ star candidate to a nearby SFR, we assumed a projected rectangular geometry in the sky for the SFR. We associated the candidate Herbig Ae/Be star to a SFR if it is positionally within the sky region covered by the SFR. We employed the SFRs from the "Study of Reflection Nebulae" by van den Bergh (1966), the "Hipparcos census of nearby $O B$ associations" by de Zeeuw et al. (1999), and the distance measurements of Lynds galactic dark nebulae by Hilton \& Lahulla (1995). In Table 1, we show the positions and extent of the SFRs employed.

The sample was complemented with two new Herbig Ae/Be star candidates (CD-38 4380 and HD 145718) from Vieira et al. (2003) associated with a SFR at $d<250$ pc. In Table 2, we present a summary of the studied stars, their coordinates and the positionally coincident SFR. The coordinates used for the Herbig Ae/Be star candidates are the coordinates of the brightest $K$ band 2MASS object in the 30" vicinity of the coordinates given by the Thé et al. catalog. In Figs. 2 and 1, we present optical $R$ band (ESO-MAMA) and $K$ band (2MASS) images of the $0.5^{\prime} \times 1^{\prime}$ field centered on the target position ${ }^{2}$.

\subsection{Observations and data reduction}

High-resolution optical spectra of the sources were obtained in April-July 2004, July-August 2007, and May 2008, using the Fiber-fed Extended Range Optical Spectrograph (FEROS; Kaufer et al. 1999) at the ESO/MPG 2.2 m telescope in la Silla Observatory $^{3}$. FEROS covers the complete optical spectral

\footnotetext{
2 Images were obtained using the Aladin software from the CDS, Strasbourg: http://aladin.u-strasbg. fr

3 http://www. ls. eso.org/lasilla/sciops/2p2/E2p2M/FEROS
} 
A. Carmona et al.: New Herbig Ae/Be stars

Table 1. Positions and extent in galactic coordinates of the star forming regions (SFR) employed.

\begin{tabular}{|c|c|c|c|c|c|c|c|}
\hline Name & Object $^{b}$ & $\begin{array}{c}11^{a} \\
{[\mathrm{deg}]}\end{array}$ & $\begin{array}{c}12 \\
{[\mathrm{deg}]}\end{array}$ & $\begin{array}{c}\mathrm{b} 1 \\
{[\mathrm{deg}]}\end{array}$ & $\begin{array}{c}\mathrm{b} 2 \\
{[\mathrm{deg}]}\end{array}$ & $\begin{array}{c}\mathrm{d} \\
{[\mathrm{pc}]}\end{array}$ & References \\
\hline Sgr R1 & $\mathrm{RN}$ & 7 & 14 & -2 & 1 & 1560 & van den Bergh (1966) \\
\hline Vul R1 & RN & 53 & 57 & 0 & 4 & 440 & van den Bergh (1966) \\
\hline Cyg OB4 & NOBA & 81 & 85 & -9 & -6 & 1000 & de Zeeuw et al. (1999) \\
\hline Cyg OB7 & NOBA & 84 & 96 & -5 & 9 & 740 & de Zeeuw et al. (1999) \\
\hline Lac OB1 & NOBA & 94 & 107 & -19 & -7 & 368 & de Zeeuw et al. (1999) \\
\hline Сер OB2 & NOBA & 96 & 108 & -1 & 12 & 615 & de Zeeuw et al. (1999) \\
\hline Cер OB6 & NOBA & 100 & 110 & -2 & 2 & 270 & de Zeeuw et al. (1999) \\
\hline Cep R2 & RN & 103 & 112 & 10 & 15 & 700 & van den Bergh (1966) \\
\hline Cep R1 & $\mathrm{RN}$ & 105 & 109 & 3 & 7 & 700 & van den Bergh (1966) \\
\hline Cер OB3 & NOBA & 108 & 113 & 1 & 7 & 960 & de Zeeuw et al. (1999) \\
\hline Cep OB4 & NOBA & 116 & 120 & 3 & 7 & 845 & de Zeeuw et al. (1999) \\
\hline Cam OB1 & NOBA & 130 & 153 & -3 & 8 & 900 & de Zeeuw et al. (1999) \\
\hline$\alpha$ Persei & NOBA & 140 & 155 & -11 & -3 & 177 & de Zeeuw et al. (1999) \\
\hline Per OB2 & NOBA & 156 & 164 & -22 & -13 & 318 & de Zeeuw et al. (1999) \\
\hline Per R1 & RN & 156 & 161 & -22 & -17 & 400 & van den Bergh (1966) \\
\hline Tau R1 & $\mathrm{RN}$ & 166 & 167 & -24 & -23 & 125 & van den Bergh (1966) \\
\hline Tau R2 & $\mathrm{RN}$ & 173 & 175 & -17 & -13 & 125 & van den Bergh (1966) \\
\hline Ori OB1 & NOBA & 197 & 215 & -26 & -12 & 500 & de Zeeuw et al. (1999) \\
\hline Mon OB1 & NOBA & 201 & 205 & -3 & 3 & 715 & de Zeeuw et al. (1999) \\
\hline Mon R1 & RN & 201 & 204 & -1 & 3 & 715 & van den Bergh (1966) \\
\hline Ori R1 & RN & 204 & 207 & -18 & -13 & 500 & van den Bergh (1966) \\
\hline Ori R2 & RN & 208 & 213 & -21 & -18 & 500 & van den Bergh (1966) \\
\hline Cma R1 & $\mathrm{RN}$ & 222 & 226 & -4 & -2 & 1315 & van den Bergh (1966) \\
\hline Col 121 & NOBA & 222 & 244 & -15 & -3 & 592 & de Zeeuw et al. (1999) \\
\hline Vela OB2 & NOBA & 255 & 270 & -15 & -2 & 410 & de Zeeuw et al. (1999) \\
\hline Trumpler 10 & NOBA & 255 & 270 & -2 & 4 & 366 & de Zeeuw et al. (1999) \\
\hline Sco OB2 -5 & NOBA & 273 & 292 & -20 & 5 & 145 & de Zeeuw et al. (1999) \\
\hline Low.Cen.Crux & NOBA & 285 & 312 & -10 & 21 & 118 & de Zeeuw et al. (1999) \\
\hline Sco OB2-4 & NOBA & 292 & 313 & -10 & 16 & 145 & de Zeeuw et al. (1999) \\
\hline Up.Cen.Lup & NOBA & 310 & 345 & 0 & 25 & 140 & de Zeeuw et al. (1999) \\
\hline Sco OB2-3 & NOBA & 313 & 337 & 5 & 31 & 145 & de Zeeuw et al. (1999) \\
\hline Sco OB2-1 & NOBA & 330 & 3 & -19 & 7 & 145 & de Zeeuw et al. (1999) \\
\hline Sco OB2-2 & NOBA & 337 & 3 & 7 & 32 & 145 & de Zeeuw et al. (1999) \\
\hline Sco R1 & RN & 346 & 2 & 13 & 23 & 160 & van den Bergh (1966) \\
\hline
\end{tabular}

Notes. ${ }^{(a)}$ The SFR is assumed to be a rectangle in the sky. The galactic coordinates 11,12 , b1, and b2 define such rectangle; ${ }^{(b)} \mathrm{RN}$ means reflection nebulae and NOBA means nearby OB association.

region (3500-9200 $\AA$ ) in one exposure with a resolution of $R \sim 45000$. One of the two fibers was positioned at the location of the target star, the second fiber was positioned at the sky. Calibration flat fields, darks frames and Th-Ar lamp calibration spectra were observed at the beginning of the night for each set of observations. The data were reduced using the FEROS-DRS pipeline provided by ESO. The typical achieved signal-to-noise ratio $(S / N)$ is over 30 . In some cases the $S / N$ achieved is of the order of 60 or larger, only in a few cases was the $S / N$ under 30 (see Table 2).

\section{Analysis}

\subsection{Spectral classification}

The spectral type and the luminosity class have been determined by careful comparison with the high-resolution $(R \sim 80000)$ spectra from the UVES Paranal Observatory Project spectral library $^{4}$ (Bagnulo et al. 2003), the ELODIE $(R \sim 42000)$ spectral library $^{5}$ (Soubiran et al. 1998), the BLUERED ${ }^{6}$ high-resolution

\footnotetext{
${ }^{4}$ http://www.sc.eso.org/santiago/uvespop/

5 http://atlas.obs-hp.fr/elodie/

${ }^{6}$ http://www . inaoep. mx/ modelos/bluered/bluered.html
}

$(R \sim 500000)$ synthetic spectral library (Bertone et al. 2008), and in the particular case of early B-type stars $^{7}$, non-LTE TLUSTY $(R \sim 300000)$ models of B-type stars (Lanz \& Hubeny 2007).

A dedicated interactive IDL-based software was developed ${ }^{8}$ to visualize the normalized target spectrum simultaneously (i.e. over-plotted) with a normalized template spectrum at a user selected spectral feature. Target and template spectra were normalized either by the median or by a polynomial fit of order 2 to the flux in the 20-100 $\AA$ windows used for the spectral comparison. Template spectra were degraded down to the FEROS resolution prior to the analysis. The comparison software allows the target or template spectrum to be shifted in wavelength such that target and template spectra had the absorption features at the same wavelength (i.e. to correct for the radial velocity difference of the star and the template). It displays the residuals in real time between the target and template spectrum (square root of the summed square of the difference between the normalized template and target spectrum), it permits calculation of equivalent widths, and it performs Gaussian fits to spectral lines.

\footnotetext{
7 TLUSTY models describe better than the BLUERED models the observed strength of the He I lines in early B-type stars.

8 Available from A. Carmona.
} 
Table 2. Studied stars, SFRs positionally coincident and summary of the observations.

\begin{tabular}{|c|c|c|c|c|c|c|c|c|c|}
\hline & $\alpha(\mathrm{J} 2000.0)$ & $\delta(\mathrm{J} 2000.0)$ & $l$ & $b$ & & $d_{\mathrm{SFR}}$ & $t_{\exp }$ & & Date(s) \\
\hline $\operatorname{Star}^{a}$ & {$\left[\begin{array}{lll}h & m & s\end{array}\right]$} & {$\left[\begin{array}{lll}0^{\circ} & 1 & \prime \prime\end{array}\right]$} & {$[\mathrm{deg}]$} & [deg] & SFR & {$[\mathrm{pc}]$} & {$[\mathrm{s}]$} & $S / N^{b}$ & [yyyy-mm-dd] \\
\hline CD-38 4380 & 082311.86 & -390701.5 & 257.32 & -1.06 & Gum Nebula & $200-240$ & 1200 & 25 & $2004-04-03$ \\
\hline WRAY 15-488 & 100148.11 & -591212.5 & 282.70 & -3.17 & ScoOB2-5 & 145 & 800 & 25 & 2004-04-04 \\
\hline WRAY 15-522 & 101212.42 & -623233.1 & 285.69 & -5.13 & ScoOB2-5 & 145 & 1500 & 25 & 2004-04-05 \\
\hline Th $35-41$ & 102540.07 & -58 2217.4 & 284.78 & -0.73 & ScoOB2-5 & 145 & 1800 & 15 & 2004-04-08 \\
\hline Hen 3-416 & 102544.51 & -583352.2 & 284.89 & -0.89 & ScoOB2-5 & 145 & 1200 & 30 & 2004-04-07 \\
\hline WRAY 15-566 & 102551.36 & -605313.2 & 286.13 & -2.86 & Low.Cen.Crux - ScoOB2-5 & $118-145$ & 1800 & 10 & 2004-04-07 \\
\hline HD 305773 & 105603.88 & -602937.6 & 289.18 & -0.74 & Low.Cen.Crux - ScoOB2-5 & $118-145$ & 700 & 100 & 2008-05-21 \\
\hline WRAY $15-770$ & 111128.49 & -630023.7 & 291.87 & -2.30 & Low.Cen.Crux - ScoOB2-5 & $118-145$ & 1200 & 10 & 2004-04-05 \\
\hline Hen 2-80 & 122223.18 & -631716.8 & 299.67 & -0.60 & Low.Cen.Crux - ScoOB2-4 & $118-145$ & 1800 & 25 & 2004-04-08 \\
\hline Hen 3-823 & 124842.39 & -595435.0 & 302.59 & 2.96 & Low.Cen.Crux - ScoOB2-4 & $118-145$ & 900 & 60 & 2008-04-21 \\
\hline Th $17-35$ & 132003.59 & -622354.0 & 306.24 & 0.29 & Low.Cen.Crux - ScoOB2-4 & $118-145$ & 1800 & 25 & 2004-04-08 \\
\hline WRAY 15-1104 & 132951.02 & -560653.7 & 308.30 & 6.36 & Low.Cen.Crux - ScoOB2-4 & $118-145$ & 1500 & 40 & 2004-04-06 \\
\hline WRAY 15-1372 & 155350.59 & -514305.1 & 329.21 & 1.59 & Up.Cen.Lup & 140 & 1500 & 30 & 2004-04-08 \\
\hline Hen $3-1121 \mathrm{~N}$ & 155809.62 & -535118.3 & 328.345 & -0.463 & $\ldots$ & - & 1500 & 70 & 2008-04-21 \\
\hline Hen 3-1121S & 155809.67 & -535134.9 & 328.342 & -0.466 & $\ldots$ & - & 1500 & 85 & 2008-04-24 \\
\hline Hen 3-1145 & 160854.69 & -393743.1 & 339.21 & 8.95 & Up.Cen.Lup & 140 & 2000 & 16 & 2004-04-08 \\
\hline WRAY 15-1435 & 161306.68 & -502320.0 & 332.36 & 0.58 & $\ldots$ & - & 1800 & 55 & 2008-04-24 \\
\hline HD 145718 & 161311.59 & -222906.6 & 352.43 & 20.44 & $\rho \mathrm{Oph}-\mathrm{Sco}$ OB2 & $110-160$ & 1000 & 20 & 2004-04-08 \\
\hline HD 152291 & 165424.20 & -403909.0 & 344.40 & 1.88 & Up.Cen.Lup & 140 & 600 & 100 & 2008-04-21 \\
\hline Hen 3-1347 & 171024.15 & -184900.7 & 4.10 & 12.26 & $\ldots$ & - & 1800 & 70 & 2007-08-04 \\
\hline WRAY 15-1651 & 171445.03 & -361838.4 & 350.27 & 1.35 & $\ldots$ & - & 2400 & 17 & 2008-05-01 \\
\hline WRAY 15-1650 & 171532.79 & -555422.7 & 334.28 & -10.07 & $\ldots$ & - & 900 & 8 & 2007-08-17 \\
\hline HD 323154 & 172302.36 & -390352.5 & 348.96 & -1.57 & $\ldots$ & - & 600 & 120 & 2007-08-04 \\
\hline WRAY 15-1702 & 172430.88 & -373427.7 & 350.35 & -0.97 & $\ldots$ & - & 1700 & 8 & 2008-04-30 \\
\hline MWC 878 & 172444.70 & -384351.4 & 349.42 & -1.65 & $\ldots$ & - & 900 & 70 & 2007-08-04 \\
\hline AS 231 & 173021.66 & -334529.6 & 354.18 & 0.17 & $\ldots$ & - & 1400 & 140 & 2004-07-31 \\
\hline Hen 3-1428 & 173502.49 & -492626.4 & 341.41 & -9.03 & $\ldots$ & - & 1200 & 50 & 2007-08-07 \\
\hline HD 320156 & 173758.51 & -352304.3 & 353.66 & -2.03 & $\ldots$ & - & 600 & 90 & 2007-08-07 \\
\hline MWC 593 & 174910.16 & -241421.2 & 4.43 & 1.75 & $\ldots$ & - & 720 & 85 & 2007-07-31 \\
\hline HD 313571 & 180107.18 & -221504.0 & 7.53 & 0.39 & $\ldots$ & - & 900 & 70 & 2007-08-02 \\
\hline MWC 930 & 182625.24 & -071317.8 & 23.65 & 2.23 & $\ldots$ & - & 2100 & 50 & 2007-08-04 \\
\hline MWC 953 & 184328.43 & -034616.9 & 28.69 & 0.05 & $\ldots$ & - & 1200 & 60 & 2007-07-31 \\
\hline AS 321 & 184704.80 & -114102.3 & 22.02 & -4.35 & $\ldots$ & - & 1500 & 75 & 2004-07-31 \\
\hline MWC 314 & 192133.97 & +145257.0 & 49.57 & 0.25 & $\ldots$ & - & 900 & 65 & 2007-08-04 \\
\hline
\end{tabular}

Notes. ${ }^{(a)}$ All sources from Table 4b of Thé et al. (1994), except for Hen 3-1145 (Table 2), Hen 2-80 (Table 4a) and CD-38 4380 and HD 145718 (Vieira et al. 2003).

(b) The $S / N$ is the average $S / N$ in the continuum close to the $\mathrm{H} \alpha$ line.

In addition, the tool allows the effect of stellar rotation onto a spectral template to be visualized. This refinement was necessary to deduce the spectral type and $v \sin (i)$ of fast rotating candidates. For this we convolved the spectral template with the 1-d normalized kernel of a rotationally broadened line.

We compared our FEROS spectra with the spectral templates, spectral feature by spectral feature by employing a window of width 20-100 $\AA$ around the central wavelength of each feature. The selected comparison spectral features were extracted primarily from the "Atlas of stellar spectra" by Ginestet et al. (1992) and the recommended features by Morgan et al. (1943) from "An atlas of stellar spectra with an outline of spectral classification”. Essentially, the procedure consisted of employing the Ginestet et al.; and Morgan et al. works to guide us in which spectral diagnostics to analyze or look at and using the UVES, ELODIE, BLUERED, or TLUSTY spectral library templates to compare the presence or absence, and the strength and shape of absorption lines to our spectra.

First, the presence or absence of certain photospheric features allowed us to constrain, in a relatively straightforward way, the spectral type up to two or three spectral subclasses. The absence of a feature sets a lower or upper limit to the spectral range, and the presence of other spectral feature(s) sets the complementary lower or upper limit to narrow the possible spectral range. Afterwards, ratios between lines (e.g., He I at $4471 \AA$ and Mg II at $4481 \AA$ ) provide an additional constraint to the spectral type range. The key to the method is to use the absence of spectral lines as an additional spectral classification criterium to the strength of the observed absorption lines. Finally, a closer comparison with the spectral library templates allowed us to narrow the classification to one spectral sub-type. Several spectral lines were used to classify each source. In Appendix 1, we describe the diagnostics that we employed for each star.

Once the spectral type was found, we determined the luminosity class. For this, we mainly relied on the diagnostics described in Ginestet et al. (1992), specifically those shown in the plates describing the effect of the luminosity for each subspectral type. In the case of M-type stars, we based the luminosity classification on the diagnostics proposed by Montes et al. (1999). In general, surface gravity diagnostics are additional spectral features to the ones used for the spectral classification. In cases where a few spectral types matched the spectra under study (e.g., in objects with large $v \sin (i))$, the template spectrum that exhibited the smallest residuals was adopted as the best match for the 
A. Carmona et al.: New Herbig Ae/Be stars
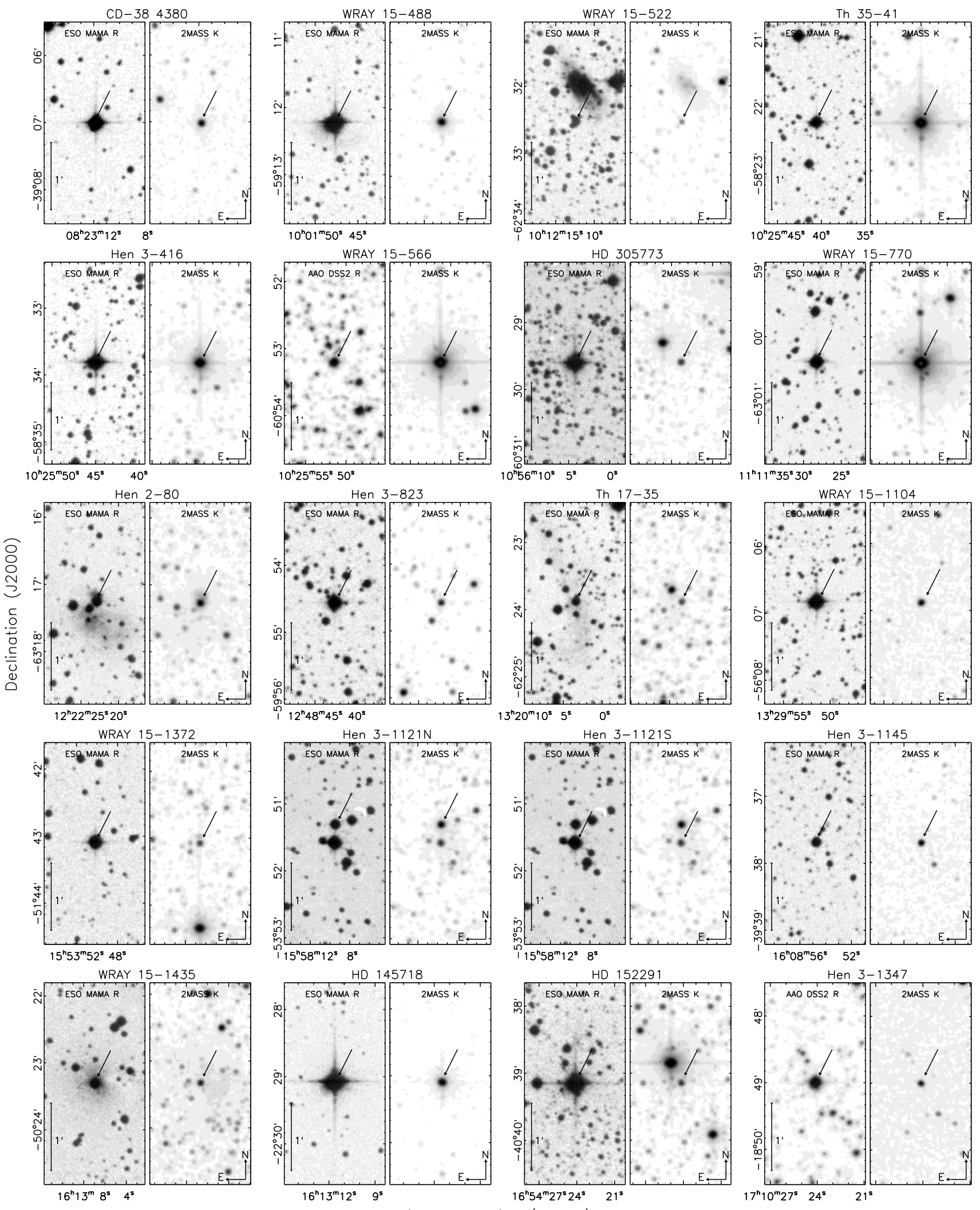

Fig. 1. Optical $\left(R\right.$ band) and near-IR $\left(K\right.$ band) images of the $0.5^{\prime} \times 1^{\prime}$ field centered on the target stars. The target is indicated by an arrow.

spectrum of the target. In Appendix 1, we describe in detail the spectral and luminosity class classification for each star individually. In Col. 2 of Table 3, we summarize the results of the spectral classification of our sample.
In Figs. 4 and 5, we present two examples of the spectral classification results. We show the spectra of the Herbig Be star WRAY 14-1435 and the B-type supergiant star Hen 3-418, together with rotationally-broadened TLUSTY models. Each 

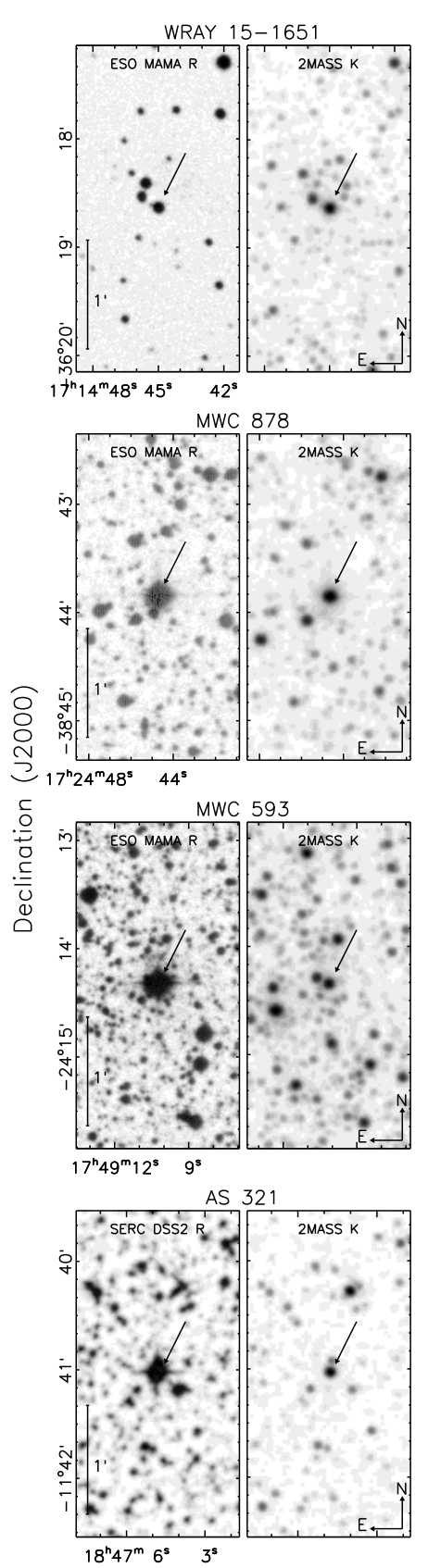
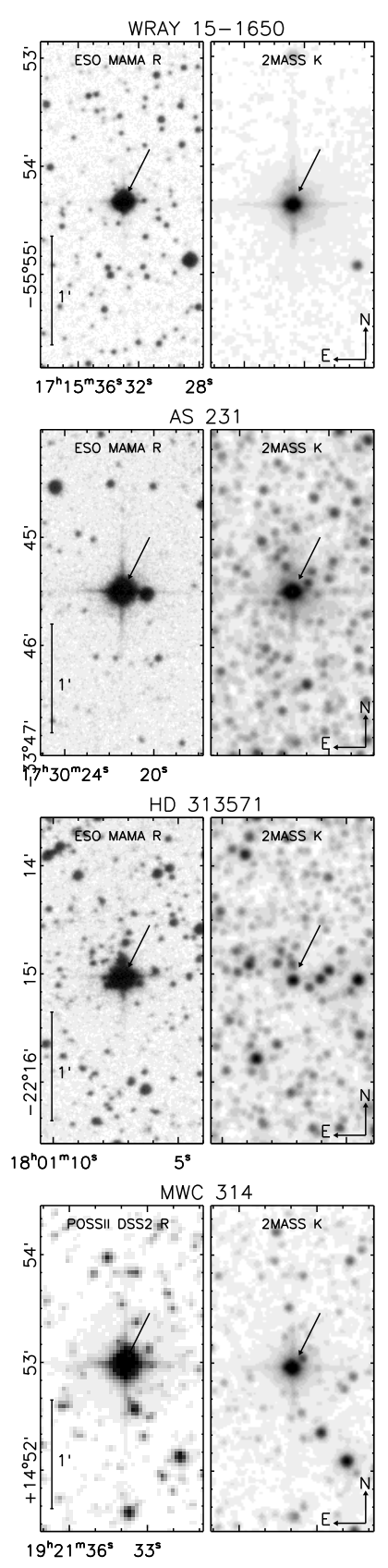
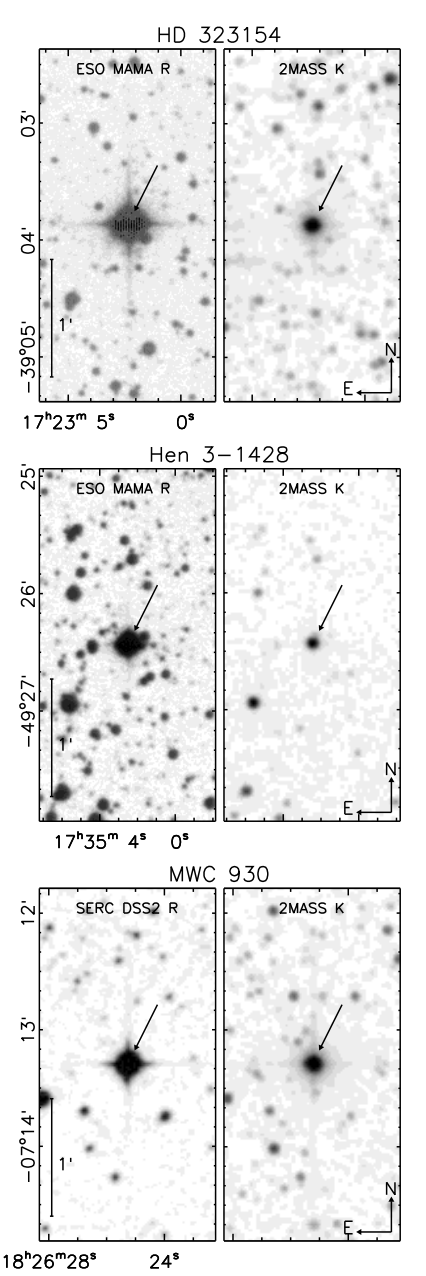
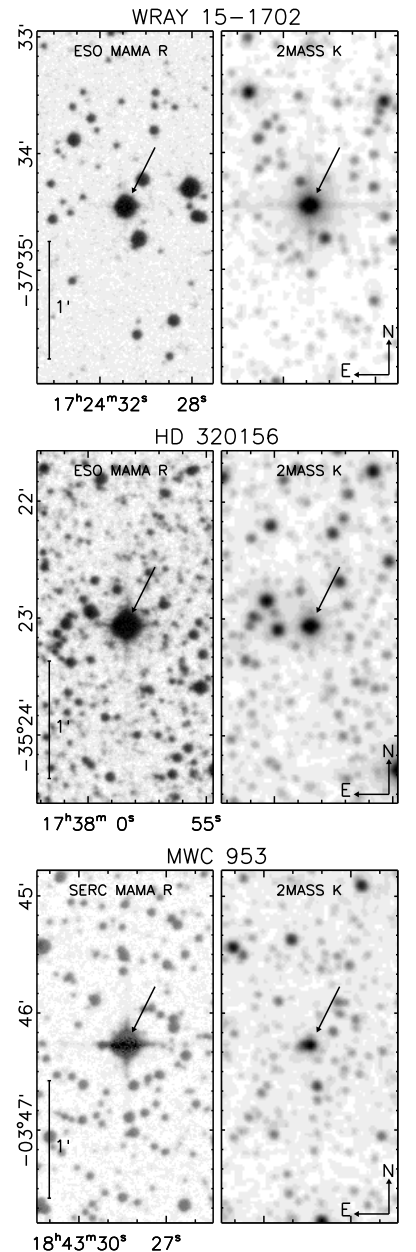

Right Ascension (J2000)

Fig. 2. Optical $\left(R\right.$ band) and near-IR $\left(K\right.$ band) images of the $0.5^{\prime} \times 1^{\prime}$ field centered on the target stars. The target is indicated by an arrow.

panel presents the FEROS spectrum of the star with three TLUSTY models. In the two panels at the top, we keep the surface gravity constant (i.e. luminosity class) and we vary $T_{\text {eff }}$. The theoretical spectrum in the middle corresponds to a TLUSTY model with $T_{\text {eff }}$ of the spectral type and luminosity class found (see Table 3). The upper and lower theoretical spectra display templates that are one spectral subclass distant in effective temperature. These panels cover wavelengths including the He I line at $4471 \AA$, the $\mathrm{Mg}$ II line at $4481 \AA$, the He II line at $4686 \AA$, and the He I line at $4388 \AA$, all important diagnostics for determining the spectral type of B-type stars. In the two panels at the bottom, we keep the $T_{\text {eff }}$ constant at a value corresponding to the spectral type found and vary the luminosity class of the theoretical spectrum from a dwarf (class V), to a giant (class III), and to a supergiant (class I). These panels cover wavelengths including the N II line at $4630 \AA$, the O II lines at 4638 and $4641 \AA$, and the C III - O II lines at $4650 \AA$, important diagnostics for establishing the luminosity class (i.e. $\log (g)$ ). The typical uncertainty in the spectral classification is one spectral subclass and 0.5 in $\log (g)$ depending on the spectral-type and $v \sin (i)$.

\subsection{Distance determination}

We estimated the distance of the sources based on spectroscopic parallaxes. We used the classical expression $V-M_{V}-A_{V}=$ $5 \log D-5$, where: $V$ is the observed $V$ magnitude, $M_{V}$ is the intrinsic absolute magnitude corresponding to the spectral type and luminosity class found and $A_{V}$ is the extinction. $A_{V}$ was calculated assuming the standard interstellar medium extinction law 
A. Carmona et al.: New Herbig Ae/Be stars
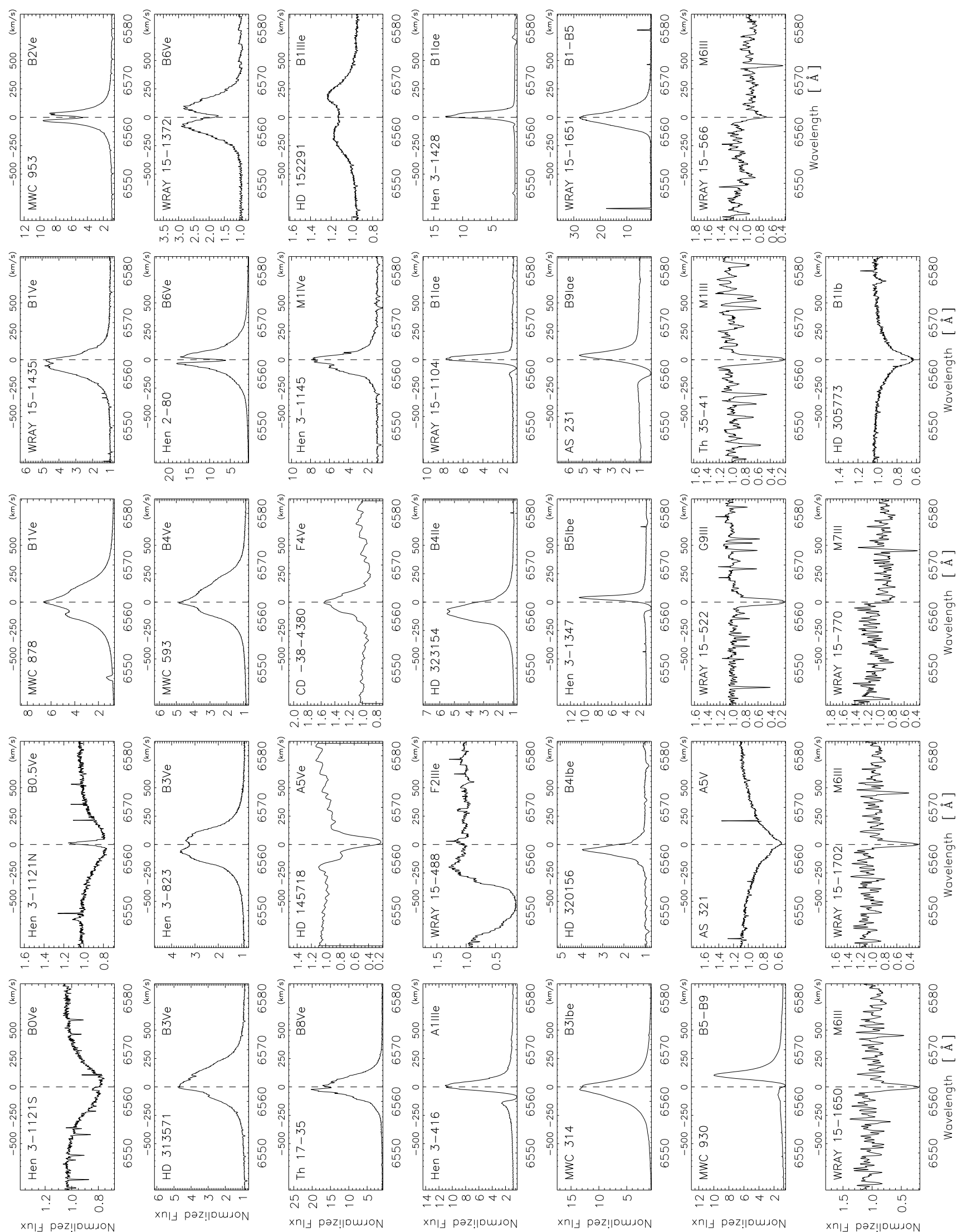

Fig. 3. Optical spectra of our sources centered on the $\mathrm{H} \alpha$ line. The spectra are barycentric and radial-velocity corrected (see Table 3). A velocity scale is given on top of each panel. 

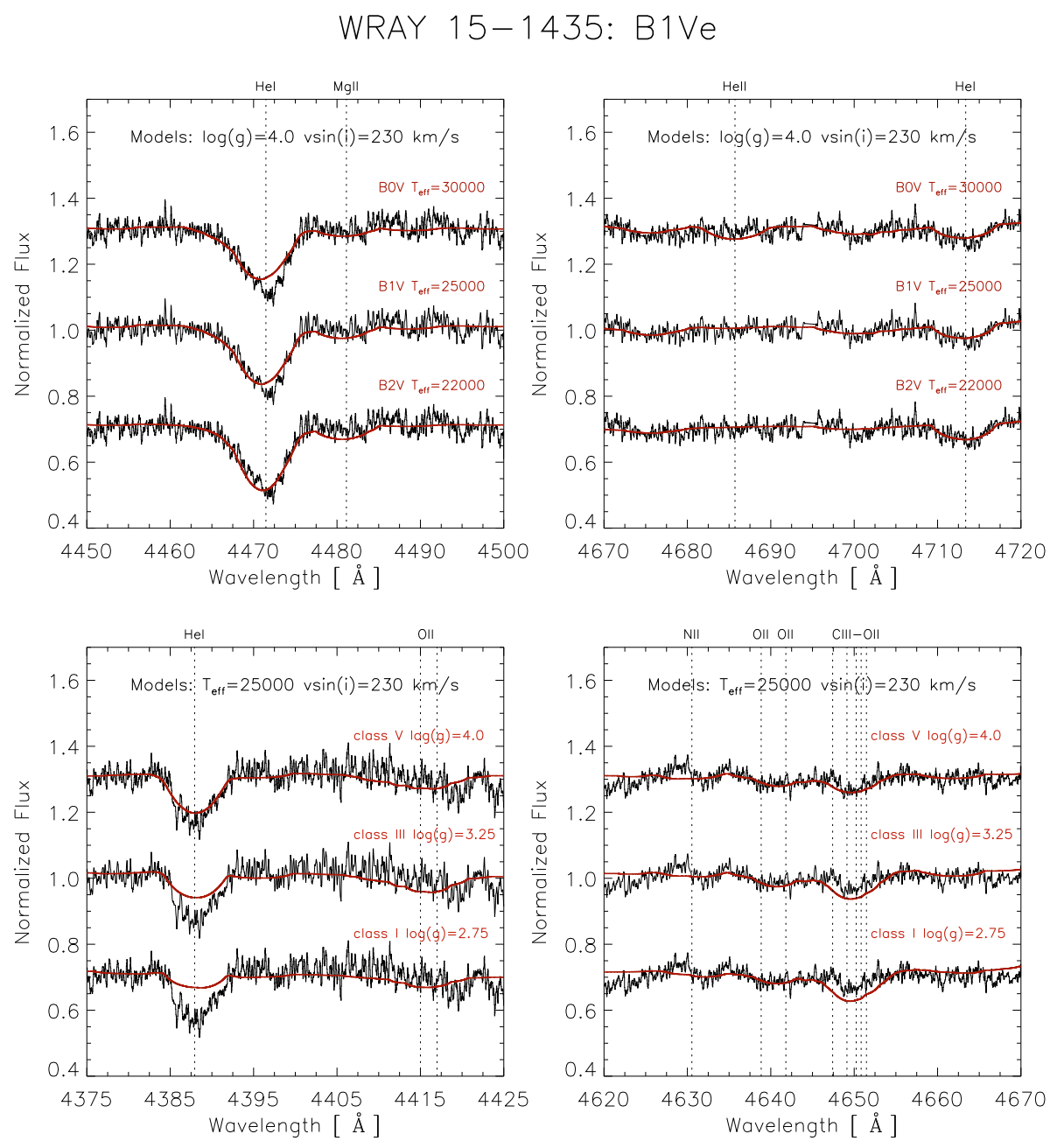

Fig. 4. Example of FEROS spectra of the Herbig Be star WRAY 15-1435 (spectral type B1Ve), together with rotationally broadened TLUSTY models (in red). The spectra are shown at the rest velocity of the star. See text for a description.

(Schultz \& Wiemer 1975) $A_{V}=3.14 E(B-V)$. For the stars with $B$ and $V$ magnitude measurements from different catalogs (i.e. different epochs) but with $R$ band measurements from the same catalog, we employed $E(B-V)=E(V-R) / 0.78$ (Schultz $\&$ Wiemer 1975). We employed the intrinsic colors and absolute magnitudes (corresponding to each spectral type and luminosity class) of Schmidt-Kaler (1982) and photometry from the literature (most notably the NOMAD catalog). The error on $M_{V}$ and $(B-V)_{0}$ was set by the uncertainty of one spectral subclass in the spectral classification. Some stars exhibited negative $E(B-V)$. This is most likely due to photometry measurements taken at different epochs. Herbig Ae/Be stars and post-main sequence supergiants show large variations in the optical, hence the need to take (quasi-)simultaneous photometry. For these sources no correction for reddening was performed. The resulting distances are given in Col. 8 of Table 3.

Given that the absolute magnitude for the luminosity class IV is not provided by the Schmidt-Kaler (1982) tables, in the case of Hen 3-1145 (spectral type M1IVe), we calculated the absolute magnitude $\left(M_{V}=7.9 \pm 0.6\right)$ from its $T_{\text {eff }}(3720 \mathrm{~K})$ and $\log (g)$ $\left(4.0 \mathrm{~g} / \mathrm{cm}^{2}\right)$ (see Appendix 1). We used the classical expressions $R=\sqrt{G M_{\star} / g}, M_{\text {bol }}=M_{\text {bol } \odot}-5 \log \left(R / R_{\odot}\right)-10 \log \left(T_{\text {eff }} / T_{\odot}\right)$, and $M_{V}=M_{\mathrm{bol}}-B C$. We assumed a mass of $0.46 M_{\odot}$ for Hen 31145 (i.e. mass of a M1V star) and a bolometric correction (BC) of -1.53 (i.e. median between the $\mathrm{BC}$ of the class $\mathrm{V}$ and class III for the M1 spectral type). The uncertainty in $M_{V}$ being largely dominated by uncertainty on the value of $\log (g)$. With an uncertainty in $\log (g)$ of 0.25 translating into an uncertainty of 0.6 mag in $M_{V}$.

\subsection{Rotational velocity}

Employing our target - template interactive spectral comparison software, in a similar way as done with the spectral classification, we compared the continuum normalized FEROS spectrum with continuum normalized rotationally broadened theoretical spectra using $T_{\text {eff }}$ and $\log (g)$ corresponding to the spectral type found for each of our stars. The template spectrum was shifted wavelength in order to account for the radial velocity of the target (see next section). We used the theoretical spectral library BLUERED and in the case of early B-type stars the non-LTE B-star models TLUSTY. We employed the $T_{\text {eff }}, \log (g)$ calibration tables of Lang et al. (1991). The rotational broadening was calculated with the IDL routines kindly provided by Bertone et al. in the BLUERED spectral library web-site. For the analysis, once the BLUERED or TLUSTY theoretical spectra were rotationally broadened, they were degraded to the FEROS resolution. For this, we used as well the Bertone et al. BLUERED IDL tools.

The projected rotational velocity uncertainty is the interval of $v \sin (i)$ values that exhibited the smallest residuals and that best fitted the width and shape of the absorption lines of the 

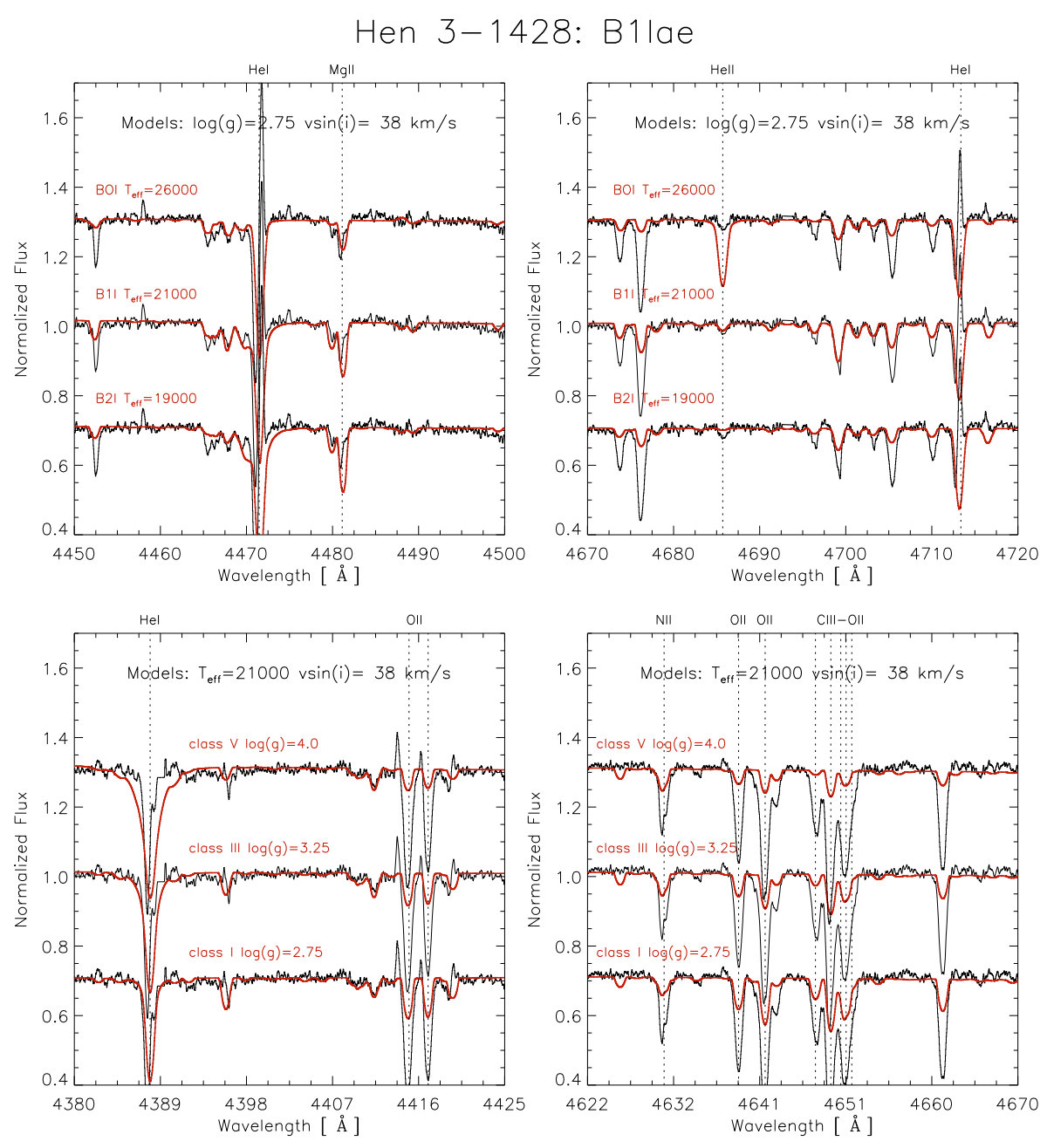

Fig. 5. Similar to Fig. 4 for the supergiant star Hen 3-1428 (spectral type B1Iae). The He I line at $4471 \AA$ displays a P-Cygni profile.

FEROS spectrum. The uncertainty of $v \sin (i)$ was typically $10 \%$, the principal source of uncertainty being the value of $T_{\text {eff }}$. This being due to the fact that for a particular spectral type a range of $T_{\text {eff }}$ exist that are consistent with it, and for each $T_{\text {eff }}$ there is a range of $v \sin (i)$ that fit the spectra. We present our results in Col. 9 of Table 3 .

\subsection{Radial velocity}

The FEROS pipeline effectuates the barycentric correction to the spectra (i.e. correction for the orbital motion of the Earth relative to the solar system barycenter). We derived the radial velocity of our targets with our interactive tool by determining the velocity shift of our FEROS spectrum with respect to a rotationally broadened BLUERED or TLUSTY synthetic spectrum using the $T_{\text {eff }}$ and $\log (g)$ of the spectral type and luminosity class found. We proceeded by first continuum-normalizing our FEROS spectrum and the synthetic spectrum, then interactively shifting the velocity of the FEROS spectrum until the center of the absorption lines of target were at the same wavelength as the absorption lines of the synthetic spectrum and the residuals between target and the synthetic spectrum were minimized. We used several regions in the spectra with strong absorption lines according to spectral type to obtain several estimations of the velocity shift. The adopted velocity shift is the average of the velocity shifts found. In the cases where the absorption lines are very weak, we employed Gaussian fits to the hydrogen or He I emission lines to constrain the radial velocity. We used the Atomic Line List v2.4 website for the wavelength calibration ${ }^{9}$. The typical error of the radial velocity is of a few $\mathrm{km} \mathrm{s}^{-1}$ but can be as large as $10-20 \mathrm{~km} \mathrm{~s}^{-1}$, depending on the spectral type and projected rotational velocity. This is particularly relevant for B stars for which the velocity shift is mostly based on the He I lines. Our results are summarized in Col. 10 of Table 3.

\subsection{Equivalent widths of selected emission lines}

Employing our spectral-comparison software, we measured the equivalent widths of selected emission lines observed in the spectra: H $\alpha$, Paschen (17), He I at $6548 \AA$, Ca II at $8498 \AA$ and $8662 \AA,[\mathrm{OI}]$ at 6301, 6365 and $8446 \AA$, [S II] at 6716 and $6731 \AA$, and [N II] at 6549 and $6585 \AA$. We present the result of our $E W$ measurements in Table 4.

In the cases where emission lines are observed inside a broad absorption line or the lines display P Cygni or inverse P Cygni profiles, we calculated individually the $E W$ of the absorption and emission component. To calculate the $E W$ of $\mathrm{P}$ Cygni profiles, we first determined the continuum using a linear interpolation of

\footnotetext{
9 Hosted by the Department of Physics and Astronomy, University of Kentucky, and maintained by Peter van Hoof Royal Observatory of Belgium. http://www . pa. uky . edu/ peter/atomic/
} 
Table 3. Established spectral type, literature photometry, estimated distance, radial and projected rotational velocity, observed H $\alpha$ and forbidden line emission.

\begin{tabular}{|c|c|c|c|c|c|c|c|c|c|c|c|}
\hline $\begin{array}{l}\text { Star } \\
\text { (1) }\end{array}$ & $\begin{array}{c}\text { Sp.Type } \\
\text { (2) }\end{array}$ & $\begin{array}{l}T_{\text {eff }} \\
{[\mathrm{K}]} \\
(3) \\
\end{array}$ & $\begin{array}{c}B \\
{[\mathrm{mag}]} \\
(4)\end{array}$ & $\begin{array}{c}V \\
{[\mathrm{mag}]} \\
(5)\end{array}$ & $\begin{array}{c}E(B-V) \\
{[\mathrm{mag}]} \\
(6)\end{array}$ & $\begin{array}{c}A_{V} \\
{[\mathrm{mag}]} \\
(7)\end{array}$ & $\begin{array}{c}d_{\text {phot }} \\
{[\mathrm{kpc}]} \\
(8)\end{array}$ & $\begin{array}{c}v \sin (i) \\
{\left[\mathrm{km} \mathrm{s}^{-1}\right]} \\
(9)\end{array}$ & $\begin{array}{c}v_{r} \\
{\left[\mathrm{~km} \mathrm{~s}^{-1}\right]} \\
(10)\end{array}$ & $\begin{array}{l}\mathrm{H} \alpha \\
(11)\end{array}$ & $\begin{array}{l}\text { F.L. } \\
\text { (12) }\end{array}$ \\
\hline \multicolumn{12}{|c|}{ Confirmed Herbig Ae/Be and T Tauri stars } \\
\hline Hen 3-1121S & B0Ve & 30000 & $11.6 \pm 0.1$ & $11.1 \pm 0.1$ & $0.85 \pm 0.14$ & $2.7 \pm 0.5$ & $3.1_{-0.8}^{+1.0}$ & $240 \pm 20$ & $-28 \pm 5$ & I & [O I] \\
\hline Hen $3-1121 \mathrm{~N}$ & $\mathrm{~B} 0.5 \mathrm{Ve}$ & 27500 & 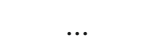 & $13.0 \pm 0.4$ & $1.3 \pm 0.7^{\star}$ & $4.1 \pm 2.3$ & $3.2_{-2 .}^{+6.8}$ & $245 \pm 20$ & $-58 \pm 5$ & I & [O I $][\mathrm{N} \mathrm{II}]$ \\
\hline MWC 878 & $\mathrm{~B} 1 \mathrm{Ve}$ & 25400 & $11.2 \pm 0.1$ & $10.8 \pm 0.1$ & $0.74 \pm 0.15$ & $2.3 \pm 0.5$ & $2.1_{-0.6}^{+0.9}$ & $\ldots$ & $-11 \pm 3^{*}$ & I & [O I $][\mathrm{N} \mathrm{II}]$ \\
\hline WRAY 15-1435 & $\mathrm{B} 1 \mathrm{Ve}$ & 25400 & $12.8 \pm 0.5$ & $12.0 \pm 0.5$ & $1.0 \pm 0.7$ & $3.2 \pm 2.2$ & $2.6_{-1.7}^{+0.0}$ & $230 \pm 20$ & $-30 \pm 10$ & I & [OI] \\
\hline MWC 953 & $\mathrm{~B} 2 \mathrm{Ve}$ & 22000 & $12.0 \pm 0.2$ & $10.9 \pm 0.1$ & $1.3 \pm 0.2$ & $4.0 \pm 0.7$ & $0.8_{-0.3}^{+0.1}$ & $<7$ & $23 \pm 2$ & II & [O I] \\
\hline HD 313571 & B3 Ve & 18700 & $10.3 \pm 0.1$ & $9.9 \pm 0.1$ & $0.57 \pm 0.15$ & $1.8 \pm 0.5$ & $0.9_{-0.3}^{+0.3}$ & $380 \pm 20$ & $-50 \pm 20$ & I & [O I] \\
\hline Hen 3-823 & $\mathrm{B} 3 \mathrm{Ve}$ & 18700 & $10.5 \pm 0.1$ & $10.4 \pm 0.1$ & $0.33 \pm 0.15$ & $1.0 \pm 0.5$ & $1.6_{-0.5}^{+0.8}$ & $310 \pm 20$ & $-10 \pm 10$ & II & [O I] \\
\hline MWC 593 & $\mathrm{~B} 4 \mathrm{Ve}$ & 17000 & $10.5 \pm 0.1$ & $10.0 \pm 0.1$ & $0.65 \pm 0.15$ & $2.1 \pm 0.5$ & $0.7_{-0.2}^{+0.5}$ & $330 \pm 30$ & $-20 \pm 10$ & I & {$[\mathrm{OI}]$} \\
\hline Hen 2-80 & B6Ve & 14000 & $14.0 \pm 0.1$ & $13.2 \pm 0.1$ & $0.97 \pm 0.15$ & $3.0 \pm 0.5$ & $1.6_{-0.4}^{-0.2}$ & $60 \pm 10$ & $-22 \pm 3$ & II & {$[\mathrm{O} \mathrm{I}][\mathrm{S} \mathrm{II}]$} \\
\hline WRAY 15-1372 & B6Ve & 14000 & $11.7 \pm 0.1$ & $11.1 \pm 0.1$ & $0.80 \pm 0.15$ & $2.5 \pm 0.5$ & $0.8_{-0.17}^{-0.4}$ & $280 \pm 20$ & $-30 \pm 10$ & II & $\ldots$ \\
\hline Th $17-35$ & $\mathrm{~B} 8 \mathrm{Ve}$ & 11900 & $13.9 \pm 0.1$ & $13.5 \pm 0.1$ & $0.58 \pm 0.15$ & $1.8 \pm 0.5$ & $2.4_{-0.6}^{-0.8}$ & $210 \pm 20$ & $-10 \pm 5$ & I & [O I] \\
\hline HD 145718 & $\mathrm{~A} 5 \mathrm{Ve}$ & 8200 & $9.3 \pm 0.1$ & $8.9 \pm 0.1$ & $0.24 \pm 0.15$ & $0.8 \pm 0.5$ & $0.17_{-0.04}^{+0.05}$ & $100 \pm 10$ & $0 \pm 3$ & IV & $\ldots$ \\
\hline CD-38 4380 & $\mathrm{~F} 4 \mathrm{Ve}$ & 6590 & $10.4 \pm 0.1$ & $10.0 \pm 0.1$ & $0.0 \pm 0.15$ & $0.0_{-00}^{+0.5}$ & $0.19_{-0.04}^{+0.04}$ & $45 \pm 4$ & $20 \pm 2$ & I & \\
\hline Hen 3-1145 & M1IVe & 3720 & $15.3 \pm 0.1$ & $14.0 \pm 0.1$ & $-0.15 \pm 0.15$ & $0.0_{-0.0}^{-0.0}$ & $0.16_{-0.04}^{+0.04}$ & $<7$ & $-2 \pm 1$ & I & [O I] \\
\hline \multicolumn{12}{|c|}{ Giant stars with emission lines that may be Herbig Ae/Be stars } \\
\hline HD 152291 & B1IIIe & 24000 & $9.0 \pm 0.1$ & $9.0 \pm 0.1$ & $0.28 \pm 0.15$ & $0.9 \pm 0.5$ & $3.1_{-09}^{+1.3}$ & $320 \pm 20$ & $-20 \pm 10$ & II & $\ldots$ \\
\hline Hen 3-416 & A1IIIJe & 9480 & $11.9 \pm 0.2$ & $10.8 \pm 0.1$ & $1.0 \pm 0.2$ & $3.3 \pm 0.7$ & $0.29_{-0.08}^{+0.12}$ & $30 \pm 5$ & $33 \pm 4$ & III, IV & {$[\mathrm{OI}]$} \\
\hline WRAY 15-488 & F2IIIe & 6870 & $10.3 \pm 0.1$ & $9.8 \pm 0.1$ & $0.11 \pm 0.15$ & $0.3 \pm 0.5$ & $0.36_{-0.07}^{+0.09}$ & $30 \pm 5$ & $-2 \pm 2$ & IV & $\ldots$ \\
\hline \multicolumn{12}{|c|}{ Giant and Supergiant stars with emission lines } \\
\hline HD 323154 & B4IIe & 15500 & $10.2 \pm 0.1$ & $9.3 \pm 0.1$ & $1.10 \pm 0.15$ & $3.4 \pm 0.5$ & $1.1_{-02}^{+0.3}$ & $55 \pm 5$ & $75 \pm 4$ & I & $\ldots$ \\
\hline WRAY $15-11$ & B1Iae & 20800 & $11.0 \pm 0.1$ & $10.8 \pm 0.1$ & $0.40 \pm 0.15$ & $1.2 \pm 0.5$ & $20_{-5}^{+7}$ & $50 \pm 5$ & $60 \pm 5$ & IV & {$[\mathrm{OI}]$} \\
\hline Hen 3-1428 & B1Iae & 20800 & $11.3 \pm 0.1$ & $10.9 \pm 0.1$ & $0.61 \pm 0.15$ & $1.9 \pm 0.5$ & $15_{-4}^{+6}$ & $38 \pm 5$ & $30 \pm 5$ & I & {$[\mathrm{O} \mathrm{I}][\mathrm{N} \mathrm{II}]$} \\
\hline MWC 314 & B3Ibe & 16200 & $11.1 \pm 0.1$ & $9.8 \pm 0.1$ & $1.45 \pm 0.15$ & $4.5 \pm 0.5$ & $1.5_{-0.3}^{-0.4}$ & $40 \pm 5$ & $65 \pm 10$ & I & {$[\mathrm{O} \mathrm{I}][\mathrm{N} \mathrm{II}]$} \\
\hline HD 320156 & B4Ibe & 14900 & $10.5 \pm 0.1$ & $9.9 \pm 0.1$ & $0.72 \pm 0.15$ & $2.3 \pm 0.5$ & $4.2_{-0.8}^{+1.3}$ & $35 \pm 5$ & $60 \pm 3$ & $\mathrm{I}$ & {$[\mathrm{O} I][\mathrm{N}$ II $]$} \\
\hline Hen 3-1347 & B5Ibe & 13600 & $11.9 \pm 0.2$ & $11.5 \pm 0.2$ & $0.56 \pm 0.28$ & $1.8 \pm 0.9$ & $10_{-4}^{+6.8}$ & $30 \pm 5$ & $-15 \pm 5$ & IV & {$[\mathrm{OI}]$} \\
\hline AS 231 & B9Iae & 10300 & $12.6 \pm 0.6$ & $10.2 \pm 0.1$ & $2.4 \pm 0.6$ & $7.5 \pm 1.9$ & $0.9_{-0.5}^{+1.3}$ & $28 \pm 5$ & $-25 \pm 5$ & IV & $\ldots$ \\
\hline \multicolumn{12}{|c|}{ Stars with strong emission line spectrum } \\
\hline WRAY 15-1651 & B1-B5 & $\ldots$ & $17.0 \pm 0.2$ & $15.8 \pm 0.2$ & $\ldots$ & $\ldots$ & $\ldots$ & $\ldots$ & & I & [O I $]$ \\
\hline MWC 930 & B5-B9 & $\ldots$ & $14.6 \pm 0.5$ & $12.8 \pm 0.5$ & $\ldots$ & $\ldots$ & $\ldots$ & $70 \pm 10$ & $10 \pm 10$ & IV & [O I $]$ \\
\hline \multicolumn{12}{|c|}{ Dwarf stars without Ho in emission } \\
\hline AS 321 & $\mathrm{~A} 5 \mathrm{~V}$ & 8200 & $12.0 \pm 0.1$ & $11.3 \pm 0.1$ & $0.60 \pm 0.23$ & $1.9 \pm 0.7$ & $0.31_{-0.09}^{+0.13}$ & $150 \pm 10$ & $-30 \pm 5$ & abs. & {$[\mathrm{O}$ I $]$} \\
\hline \multicolumn{12}{|c|}{ Giant and Supergiant stars without Ho in emission } \\
\hline WRAY 15-522 & G9III & 4820 & $14.3 \pm 0.6$ & $13.4 \pm 0.6$ & $-0.03 \pm 0.8$ & $0.0_{-00}^{+2.6}$ & $3.3_{-23}^{+7.7}$ & $<7$ & $-9 \pm 2$ & abs. & [O I $]$ \\
\hline Th $35-41$ & M1III & 3720 & $14.5 \pm 0.1$ & $13.1 \pm 0.6$ & $-0.17 \pm 0.6$ & $0.0_{-00}^{+1.0}$ & $5.1_{-26}^{-5.3}$ & $13 \pm 4$ & $15 \pm 2$ & abs. & $\ldots$ \\
\hline WRAY 15-566 & M6III & 3240 & $16.0 \pm 0.4$ & $14.2 \pm 0.8$ & $0.31 \pm 0.90$ & $1.0 \pm 2.8$ & $4.6_{-35}^{+13.6}$ & $<7$ & $29 \pm 2$ & abs. & $\ldots$ \\
\hline WRAY 15-1650 & M6III & 3240 & $13.5 \pm 0.5$ & $11.9 \pm 0.2$ & $0.13 \pm 0.54$ & $0.4 \pm 1.7$ & $2.1_{-1.1}^{+3.5}$ & $<7$ & $-22 \pm 2$ & abs. & $\ldots$ \\
\hline WRAY 15-1702 & M6III & 3240 & $13.9 \pm 0.2$ & $12.6 \pm 0.2$ & $-0.24 \pm 0.29$ & $0.0_{-0.0}^{+0.1}$ & $3.4_{-0.4}^{+0.1}$ & $<7$ & $-26 \pm 2$ & abs. & $\ldots$ \\
\hline WRAY 15-770 & M7III & 3150 & $16.5 \pm 1.0$ & $15.2 \pm 1.0$ & $-0.16 \pm 1.4$ & $0_{-0}^{+3.90}$ & $11_{-9.6}^{+6.4}$ & $<7$ & $23 \pm 3$ & abs. & $\ldots$ \\
\hline HD 305773 & $\mathrm{~B} 1 \mathrm{Ib}$ & 20800 & $9.2 \pm 0.1$ & $9.1 \pm 0.1$ & $0.27 \pm 0.15$ & $0.8^{-0} \pm 0.5$ & $6.6_{-1.3}^{+1.6}$ & $27 \pm 5$ & $-2 \pm 1$ & abs. & $\ldots$ \\
\hline
\end{tabular}

Notes. Column (3): $T_{\text {eff }}$ from Lang et al. 1991 (adapted from Schmidt-Kaler 1982). Columns (4) and (5): photometry in the $B$ and $V$ bands taken from the NOMAD catalog, except for Hen 2-80, Th 17-35, and CD-38 4380 where they are from Vieira et al. (2003) and Hen 3-1121N where they are from the GSC 2.3.2. Columns (6) and (7): Schmidt-Kaler (1982) intrinsic colors and absolute magnitudes of were used to calculate $E(B-V)$ and the distance. The absolute magnitudes for the M6III and M7III stars are absent from the Schmidt-Kaler (1982) tables. We deduced them by linear extrapolation of the $M_{V}$ of the M3III and M4III spectral types. Column (6): ${ }^{\star}$ in the case of Hen 3-1121N the $R=12.1 \pm 0.4$ band magnitude was used to derive $E(B-V)$ using $E(B-V)=E(V-R) / 0.78$. Column (7): no correction for extinction was applied to sources with $E(B-V)<0$. Columns (9) and (10): ${ }^{*}$ when the spectra are dominated by emission lines and almost no absorption lines are observed no constrain for $v \sin (i)$ is given and the $v_{r}$ estimate is based on the averaged velocity shift of emission lines (principally He I). Column (11): the type of the H $\alpha$ profile is given following the classification of Reipurth et al. (1996) and Vieira et al. (2003): type I profiles are symmetric without, or with only very shallow absorption features; type II profiles are double-peaked with the secondary peak having more than half of the strength of the primary; type III profiles are double-peaked, with the secondary peak having less than half the strength of the primary; type IV profiles have P Cygni line characteristics. Column (12): F.L. means forbidden lines. [O I] refers to the detection of [O I] lines at 6301, 6365, or 8446 ^⿱ [ [S II] refers to the [S II] line at 6716 and $6731 \AA$; [ [N II] refers to the lines at 6549 and $6585 \AA$. 
Table 4. Equivalent widths of $\mathrm{H} \alpha$, He I, Ca II, and forbidden emission lines observed in the spectra.

\begin{tabular}{|c|c|c|c|c|c|c|c|c|c|c|c|c|}
\hline Star & \multirow[t]{2}{*}{$\begin{array}{c}\mathrm{H} \alpha \\
(6563 \AA) \\
\end{array}$} & \multirow[t]{2}{*}{$\begin{array}{c}\mathrm{P}(17) \\
(8467 \AA)\end{array}$} & $\begin{array}{c}\text { He I } \\
(6548 \AA)\end{array}$ & \multicolumn{3}{|c|}{$\begin{array}{c}{[\mathrm{O} \mathrm{I}]} \\
(6301 \AA, 6365 \AA, 8446 \AA)\end{array}$} & \multicolumn{2}{|c|}{$\begin{array}{c}{[\mathrm{S} \text { II }]} \\
(6716 \AA \AA ⿻ 丿 𠃍 一\end{array}$} & \multirow{2}{*}{\multicolumn{2}{|c|}{$\begin{array}{c}{[\mathrm{N} \text { II }]} \\
\left(6549 \AA \AA^{\circ}, 6585 \AA\right)\end{array}$}} & \multicolumn{2}{|c|}{$\begin{array}{c}\text { Ca II } \\
(8498 \AA, 8662 \AA)\end{array}$} \\
\hline \multicolumn{9}{|c|}{ Confirmed Herbig Ae/Be and T Tauri stars } & & & & \\
\hline Hen $3-1121 \mathrm{~S}$ & $\mathrm{EIA}_{-01}^{+2.8}$ & $(0.1)$ & +0.8 & -0.02 & $(0.01)$ & $(0.05)$ & $(0.01)$ & $(0.01)$ & +0.01 & $(0.01)$ & $(0.01)$ & $(0.0)$ \\
\hline Hen $3-1121 \mathrm{~N}$ & $\mathrm{EIA}_{-0 .}^{+0.1}$ & $(0.1)$ & +0.8 & -0.03 & $(0.02)$ & $(0.08)$ & $(0.01)$ & $(0.01)$ & -0.04 & -0.13 & $(0.1)$ & $(0.0)$ \\
\hline MWC 878 & -54 & -2.0 & -3.5 & -0.12 & $(0.01)$ & -8.3 & $(0.01)$ & $(0.01)$ & -0.5 & -1.6 & -7.1 & -8.1 \\
\hline WRAY 15-1435 & -20 & -1.7 & +0.6 & -0.02 & $(0.02)$ & -4.1 & -0.01 & $(0.01)$ & $(0.01)$ & $(0.02)$ & -0.3 & -0.5 \\
\hline MWC 953 & -32 & -3.5 & +0.3 & -0.01 & $(0.02)$ & -2.2 & $(0.01)$ & $(0.01)$ & +0.03 & +0.1 & -0.4 & -0.7 \\
\hline HD 313571 & -34 & -2.1 & -0.3 & -0.01 & $(0.02)$ & -4.8 & $(0.02)$ & $(0.01)$ & +0.01 & $(0.01)$ & -1.3 & -0.1 \\
\hline Hen 3-823 & -25 & -1.4 & +0.6 & $(0.01)$ & $(0.03)$ & -1.4 & $(0.01)$ & $0.01)$ & $(0.01)$ & $(0.01)$ & $(0.1)$ & $(0.1)$ \\
\hline MWC 593 & -35 & -1.3 & -0.2 & -0.01 & $(0.03)$ & -3.7 & $(0.01)$ & $(0.01)$ & (0.01) & $(0.02)$ & -0.6 & $(0.1)$ \\
\hline Hen 2-80 & -150 & -1.8 & +0.6 & -9.9 & -3.4 & -16 & -0.1 & -0.2 & -0.06 & -0.2 & -0.2 & -0.2 \\
\hline WRAY 15-1372 & -15 & $(0.2)$ & $(0.02)$ & $(0.1)$ & $(0.1)$ & (0.6) & $(0.01)$ & $(0.03)$ & $(0.01)$ & $(0.1)$ & $(0.3)$ & $(0.6)$ \\
\hline Th $17-35$ & -90 & (0.6) & +0.2 & -0.3 & -0.4 & -5.3 & -0.1 & -0.1 & -0.07 & -0.2 & -0.1 & -0.2 \\
\hline HD 14 & $\mathrm{PC}_{-0.2}^{+2.4}$ & (0.6) & $(0.1)$ & $(0.2)$ & $(0.1)$ & +0.5 & $(0.01)$ & $(0.01)$ & $(0.3)$ & $(0.0)$ & +0.6 & +1.4 \\
\hline CD-38 4380 & $\mathrm{EIA}_{-3.0}^{+1.2 .0}$ & (0.6) & $(0.03)$ & $(0.1)$ & $(0.2)$ & +0.5 & $(0.1)$ & $(0.02)$ & $(0.01)$ & $(0.3)$ & +0.7 & +1.4 \\
\hline Hen 3-1145 & -30 & $(0.1)$ & -0.5 & -0.5 & $(0.3)$ & $(0.02)$ & +0.2 & $(0.04)$ & $(0.02)$ & $(0.05)$ & -0.5 & -0.5 \\
\hline \multicolumn{13}{|c|}{ Giant stars with emission lines that may be Herbig Ae/Be stars } \\
\hline HD 152291 & -3.4 & -0.8 & +0.2 & $(0.01)$ & $(0.02)$ & $(0.05)$ & $(0.01)$ & & $(0.01)$ & $(0.01)$ & -0.5 & $(0.1)$ \\
\hline Hen 3 & $\mathrm{PC}_{-27}^{+0.5}$ & $(1.0)$ & +0.2 & -0.4 & -0.2 & & $(0.1)$ & & $(0.01)$ & $(1)$ & -5.3 & -9 \\
\hline WRAY $15-488$ & $\mathrm{PC}_{-0.8}^{+7.2}$ & +1.0 & $(0.06)$ & $(0.1)$ & $(0.1)$ & +0.6 & +0.1 & $(0.1)$ & $(0.01)$ & $(0.3)$ & +1.2 & +2.0 \\
\hline \multicolumn{13}{|c|}{ Giants and Supergiants with emission lines } \\
\hline HD 323154 & -26 & -0.9 & -2.0 & $(0.01)$ & $(0.01)$ & $(0.02)$ & $(0.05)$ & $(0.01)$ & $(0.01)$ & -0.07 & -0.8 & -0.9 \\
\hline WRAY $15-1104$ & $\mathrm{PC}_{-10}^{+0.2}$ & +0.5 & $\mathrm{PC}_{-0.4}^{+0.4}$ & $(0.2)$ & -0.02 & -0.7 & $(0.01)$ & $(0.01)$ & $(0.01)$ & $(0.3)$ & $(0.5)$ & $(0.5)$ \\
\hline Hen 3-1428 & -18 & $\mathrm{PC}_{-0.04}^{+0.25}$ & -1.4 & -0.8 & -0.3 & -4.0 & $(0.02)$ & -0.05 & -0.15 & $\mathrm{PC}_{-0.9}^{+0.2}$ & $(0.1)$ & $(0.1)$ \\
\hline MWC 314 & -118 & -2.4 & -6.1 & -0.01 & $(0.01)$ & -4.7 & -0.06 & $(0.03)$ & -0.04 & -0.17 & -13 & -19 \\
\hline HD 320156 & -7.8 & -0.7 & 0.3 & $(0.02)$ & $(0.03)$ & $\mathrm{PC}_{-0.05}^{+0.2}$ & $(0.02)$ & $(0.02)$ & +0.1 & $\mathrm{PC}_{+0.03}^{-0.15}$ & -8 & -14 \\
\hline Hen 3-1347 & $\mathrm{PC}_{-12}^{+0.8}$ & +0.1 & 0.2 & -0.3 & -0.1 & -0.7 & $(0.05)$ & $(0.04)$ & $(0.01)$ & $\begin{array}{l}+0.03 \\
+0.2\end{array}$ & +0.5 & $\mathrm{EIA}_{-0.2}^{+0.7}$ \\
\hline AS 231 & $\mathrm{PC}_{-14}^{+1.4}$ & +0.7 & 0.2 & $(0.01)$ & $(0.02)$ & +0.8 & +0.01 & +0.03 & (0.03) & $(0.01)$ & +1.3 & +2.0 \\
\hline \multicolumn{13}{|c|}{ Unclassified stars with strong emission line spectrum } \\
\hline WRAY 15-1651 & & -1.2 & $(0.6)$ & -0.90 & -0.1 & -7.4 & -0.1 & -0.12 & $(0.06)$ & $(0.02)$ & -1.5 & -1.7 \\
\hline MWC 9 & $\mathrm{PC}_{-42}^{+0.1}$ & $\mathrm{PC}_{-1.0}^{+0.7}$ & +0.2 & -0.02 & $(0.01)$ & $\mathrm{PC}_{-1.5}^{+1.5}$ & $\mathrm{PC}_{-0.1}^{+0.2}$ & $(0.04)$ & -0.04 & $(0.01)$ & $\mathrm{PC}_{-5.1}^{+1.7}$ & $\mathrm{PC}_{-6.6}^{+2.0}$ \\
\hline \multicolumn{13}{|c|}{ Dwarf stars without Ho in emission } \\
\hline AS 321 & +5.7 & $(0.1)$ & $(0.03)$ & -0.02 & $(0.01)$ & +0.6 & $(0.01)$ & $(0.01)$ & $(0.01)$ & $(0.01)$ & +0.8 & +1.8 \\
\hline \multicolumn{13}{|c|}{ Giant and Supergiant stars without Ho in emission } \\
\hline WRAY $15-522$ & +1.2 & +0.2 & $(0.03)$ & -0.2 & $(0.1)$ & $(0.1)$ & +0.1 & $(0.2)$ & $(0.01)$ & $(0.1)$ & +1.3 & +2.1 \\
\hline Th $35-41$ & +1.2 & +1.4 & -0.15 & $(0.1)$ & (0.1) & $(0.2)$ & $(0.5)$ & $(0.4)$ & $(0.01)$ & $(0.2)$ & +2.2 & +2.7 \\
\hline WRAY $15-5$ & +0.4 & $(0.06)$ & -0.15 & $(0.3)$ & (0.1) & (0.1) & (0.6) & $(0$. & $(0.1)$ & $(0.2)$ & +0.6 & +0.6 \\
\hline WRAY $15-1650$ & +1.0 & +0.6 & $(0.01)$ & $(0.2)$ & (0.1) & $(0.2)$ & $(0.5)$ & $(0.2)$ & $(0.1)$ & $(0.3)$ & +1.3 & +2.1 \\
\hline WRAY $15-1702$ & +0.8 & +0.8 & $(0.02)$ & $(0.1)$ & $(0.1)$ & $(0.3)$ & $(0.5)$ & $(0.5)$ & $(0.1)$ & $(0.3)$ & +1.1 & +2.0 \\
\hline WRAY 15-770 & +0.5 & $(0.05)$ & $(0.04)$ & $(0.2)$ & $(0.1)$ & $(0.1)$ & (1.0) & $(1.0)$ & $(0.1)$ & $(0.1)$ & $\mathrm{EIA}_{-0.1}^{+0.4}$ & $\mathrm{PC}_{+0.4}^{-0.3}$ \\
\hline HD 305773 & +2.0 & $(0.07)$ & +0.7 & $(0.05)$ & $(0.01)$ & $(0.05)$ & $(0.01)$ & $(0.02)$ & $(0.01)$ & $(0.05)$ & $(0.1)$ & $(0.1)$ \\
\hline
\end{tabular}

Notes. In the cases where emission lines are observed inside a broad absorption line, we give the $E W$ of the absorption and emission component and write EIA meaning "Emission Inside Absorption". In the case of P Cygni and inverse P Cygni profiles are noted by PC and the equivalent width $(E W)$ of the absorption and emission component are given. For the stars displaying Paschen emission, the Ca II lines at 8498 and $8662 \AA$ are blended with the Paschen(16) and Paschen(13) respectively. For these sources the $E W$ of Ca II line at 8498 and $8662 \AA$, is the $E W$ measured at the position of the Ca II line minus the $E W$ of the Paschen(17) and Paschen(14) lines respectively. Upper limits are displayed in parenthesis.

the continuum from nearby regions (right and left) outside the line. Then employing the standard $E W$ formula we calculated the $E W$. For the blueshifted absorption part, we integrated from the wavelength where the continuum and left wing of the absorption line are similar (beginning of the absorption line), up to the wavelength where the right absorption wing crosses the continuum (end of the absorption line). For the redshifted emission part, we integrated from the wavelength where the emission left wing crosses the continuum (beginning of the emission line) up to the wavelength where the continuum and right wing of the emission line are similar (end of the emission line).
For the stars where Paschen (16) and Paschen (13) emission lines are blended with Ca II emission at 8498 and $8662 \AA$, we calculated the $E W$ of $\mathrm{Ca}$ II lines by subtracting to the $E W$ measured at the position of the Ca II line the $E W$ of the Paschen (17) and Paschen (14) lines respectively.

\section{Results and discussion}

The results of our FEROS spectroscopy campaign are summarized in Tables 3 and 4 . Table 3 describes the established spectral types, the estimated distance, the measured radial and projected 
rotational velocity, the type of the $\mathrm{H} \alpha$ profile observed according to the classification of Reipurth et al. (1996) and a summary of important forbidden emission lines observed. Table 4 displays the $E W$ measurements of selected emission lines in the spectrum.

From our total sample of 34 candidates, 13 sources are confirmed as Herbig Ae/Be stars ( 40\%). These objects display $\mathrm{H} \alpha$ in emission and have spectral types with luminosity class V. They are mostly Herbig Be stars. A large fraction of them (10 objects, $\sim 80 \%$ ) display forbidden line emission, in particular [OI] lines (see Table 4). Ca II emission lines at $8500 \AA$ are observed in $\sim 50 \%$ of them (6 sources). CD-38 4380 and HD 145718, the two new Herbig Ae/Be candidates from Vieira et al. (2003), are confirmed as Herbig Ae/Be stars. We observe (see Fig. 3) that, in general, for Herbig Ae/Be stars the $\mathrm{H} \alpha$ line is centered close to the velocity of the star and that its width is broader than the $\mathrm{H} \alpha$ line observed in the supergiant stars of our sample. Most notable exceptions are WRAY 15-1435 and HD 145718 where $\mathrm{H} \alpha$ is observed shifted with respect to the star's velocity and Hen 3-1121 S\&N in which $\mathrm{H} \alpha$ is narrow.

From the subsample of 16 candidates positionally coincident with nearby SFRs, 6 sources are confirmed as Herbig Ae/Be stars. From these, two sources, CD-38 4380 and HD 145718, are at distances closer than $250 \mathrm{pc}$. This provides further evidence for the association of CD-38 4380 with the Gum Nebula and of HD 145718 with Sco OB2. The remaining 4 sources, Hen 2-80, Hen 3-823, Th 17-35, and WRAY 15-1372, are at distances greater than $700 \mathrm{pc}$. They are not members of nearby SFRs.

From the subsample of 18 "isolated" candidates (i.e. candidates not known to be associated to a nearby SFRs), we confirm 7 as Herbig Ae/Be stars: Hen 3-1121 N, Hen 3-1121 S, WRAY 15-1435, MWC 878, MWC 593, HD 313571, and MWC 953. All of them have distances greater than $700 \mathrm{pc}$.

For the 11 confirmed Herbig Ae/Be stars with distances greater than $700 \mathrm{pc}$, we searched for Spitzer $8 \mu \mathrm{m}$ images and, using the SIMBAD database, for objects characteristic of SFRs in their $20^{\prime}$ vicinity (e.g., HII regions, molecular clouds, dark nebulae, maser emission, etc.). We wanted to check whether our distant Herbig Ae/Be stars are truly "isolated" sources located in empty regions, or whether they may be associated with distant SFRs. We found that seven sources (Hen 2-80, Hen 3-1121 N\&S, HD 313571, MWC 953, WRAY 15-1435, and Th 17-35) are inside or close $\left(<5^{\prime}\right)$ to regions with extended IR emission (see Fig. 6) and that in their $20^{\prime}$ vicinity there are objects characteristic of SFRs. As we do not have constrains on the distance of the extended IR emission, it is difficult to establish whether our sources are inside the emission region or if they are background or foreground sources. However, as our stars are young, we find it is likely that they are inside these IR emission regions and speculate that these regions may be distant SFRs. These regions are interesting for follow-up studies of their stellar content. We remark that in the Spitzer images, three sources (WRAY 15-1435, HD 313571, Hen 2-80) are inside or have a nearby bright IR nebulosity. This further confirms their young nature. Two sources MWC 878 and WRAY 15-1372 do not have nearby extended IR-emission nor nearby astronomical sources characteristic of SFRs. They may be a case of "isolated" Herbig Ae/Be stars. We note that Spitzer data were not available for MWC 593 and Hen 3-823. In Appendix 2, we present the Spitzer $8 \mu \mathrm{m}$ images and discuss each source individually.

Now, let us return to the discussion of the results of the complete sample. One candidate, Hen 3-1145, is found to be an early M-type emission-line PMS star. Li in absorption is observed towards this object confirming that it is a young classical
$\mathrm{T}$ Tauri star. The spectroscopic parallax distance of $160_{-40}^{+50} \mathrm{pc}$ strongly suggest its association to the Upper Centaurus Lupus SFR. Merín et al. (2008) observed this object with Spitzer and associated the source with the Lupus III dark cloud ${ }^{10}$.

Three sources, HD 152291, Hen 3-416, and WRAY 15-488 have spectral types $\mathrm{B}, \mathrm{A}$, and $\mathrm{F}$ respectively, luminosity class III and display $\mathrm{H} \alpha$ and forbidden line emission (see Tables 3 and 4). In these objects it is not entirely clear whether they are in the pre- or post-main sequence evolutionary phase. Given that young early type stars are bright, they are able to dissipate their environment relatively early. It could be that HD 152291, Hen 3-416, and WRAY 15-488 are young stars still under contraction that have become optically visible. For that reason they display the signatures of low gravity, thus a luminosity class III. However, given that they could be post-main-sequence stars as well, we classified them in the separate category "Giant stars that may be Herbig Ae/Be stars". The estimated distance can perhaps give some hints about the evolutionary stage of these sources. In the case of HD 152291 the estimated distance of $3 \mathrm{kpc}$ is not consistent with the association with the SFR Upper Centaurus Crux ( $d \sim 140 \mathrm{pc}$ ). Therefore, HD 152291 may be a background giant star. Furthermore, the high value of $v \sin (i)\left(320 \pm 20 \mathrm{~km} \mathrm{~s}^{-1}\right)$ measured in HD 152291 makes it plausible that HD 152291 is a classical Be star, i.e. an evolved object spinning near its breakup velocity, surrounded by a gaseous disk consisting of matter lost by the central star. In the case of Hen 3-416, the estimated distance of $290_{-80}^{+110} \mathrm{pc}$ does not provide strong evidence of the association to the SFR Scorpius OB 2-5 ( $d \sim 140 \pm 20-30 \mathrm{pc}$, Preibisch \& Mamajek 2008). But it might be that Hen 3-416 is a young star located at the very extreme end of the Scorpius SFR; in this case Hen 3-416 is likely to be a Herbig Ae/Be star. In the case of WRAY 15-488, the estimated distance of $360_{-70}^{+90} \mathrm{pc}$ is more than $200 \mathrm{pc}$ away than the SFR Scorpius OB 2-5. It is likely that the source is not associated to this SFR. Present data do not permit us to conclude convincingly that WRAY 15-488 is a young star.

Seven of our 34 sources (20\%) are post- main sequence supergiant stars with $\mathrm{H} \alpha$ in emission. These seven objects are not members of the Herbig Ae/Be stellar group. High-resolution spectra were able to reveal the spectral diagnostics needed to establish their evolved state. All of them display $\mathrm{H} \alpha$ in emission, and forbidden line emission was observed in five cases (WRAY 15-1104, Hen 3-1428, MWC 314, HD 320156, and Hen 3-1347). These objects are examples of post-main sequence $\mathrm{B}[\mathrm{e}]$ stars. Almost all of them display P-Cygni Balmer emission line profiles or blueshifted $\left(50-150 \mathrm{~km} \mathrm{~s}^{-1}\right) \mathrm{H} \alpha$ emission. This suggests the presence of strong winds. We observe (see Fig. 3) that, in general, the width of their $\mathrm{H} \alpha$ line is narrower than the $\mathrm{H} \alpha$ emission line observed in the Herbig Ae/Be stars of our sample. Although not in the direction of our goal of finding young stars, these objects are very interesting because they are examples of $\mathrm{B}[\mathrm{e}]$ supergiants with IR excess.

Two sources (WRAY 15-1651, MWC 930) display rich emission line spectra. Their spectra exhibit strong veiling, likely due to accretion. For these sources we were unable to derive

\footnotetext{
${ }_{10}$ Merín et al. (2008) detected emission at 24, 70, and $160 \mu \mathrm{m}$ in Hen 3-1145 with Spitzer. From the spectral energy distribution slope computed from the $K$-band to $24 \mu \mathrm{m}$, they classified the star as Class II. The low IR excess led those authors to classify the source as an "anemic" disk: it displays a $24 \mu \mathrm{m}$ flux typical of Class III sources, but $70 \mu \mathrm{m}$ excess comparable to a classical T Tauri star. Objects such as Hen 3-1145 appear to be extremely rare. They are the so-called cold disks that are interpreted as optically thick disks with large inner holes of several to tens of AU (Calvet et al. 2005; Brown et al. 2007).
} 
their luminosity class because of the nearly complete absence of photospheric absorption lines in their optical spectra. We assume they are bona-fide young stars, but we were not able to constrain their distances.

One source (AS 321) is an A-type main sequence star without emission lines. This object is located at a distance $\sim 300 \mathrm{pc}$ and it is not associated with a known nearby SFR. It might be a field A star. The lack of emission can be understood trough two scenarios. In the first scenario the star is a main-sequence star, and the measured IRAS excess is due to a debris disk surrounding the star (i.e. a Vega-like star). In another scenario, AS 321 is a young Herbig Ae star, but due to the intrinsic variability of accretion, the $\mathrm{H} \alpha$ line is absent because we observed AS 321 during a period of low accretion activity. We should note that AS 321 does exhibit [O I] forbidden line emission at $6300 \AA$ and this could be due to the presence of either a disk or an outflow ${ }^{11}$.

Finally, seven of our 34 sources (20\%) are giant (6) and supergiant (1) stars without $\mathrm{H} \alpha$ in emission (WRAY 15-522, Th 35-41, WRAY 15-566, WRAY 15-1650, WRAY 15-1702, WRAY 15-770, HD 305773). They are rejected as Herbig Ae/Be stars because they are evolved objects. Most of them have a late spectral type (i.e. M). Their distances indicate that they are fieldbackground stars. Since most of these stars originate from surveys of emission-line stars, the lack of $\mathrm{H} \alpha$ in emission was not expected. As in the case of AS 321, there is always the possibility of spectral variability of the $\mathrm{H} \alpha$ line. However, in some of these cases, the target may also simply have been misidentified, as the accuracy of coordinates in the catalogs from which these targets were selected is low. We selected the brightest $K$-band source in the vicinity of the coordinates given in the The et al. (1994) cata$\log$, but it may be that the emission line-star is a fainter IR source in the field.

\section{Summary and conclusions}

We obtained high-resolution optical spectroscopy of 34 candidates to Herbig Ae/Be stars with unknown or poorly constrained spectral types from the Thé et al. (1994) catalog and two candidates from Vieira et al. (2003). We observed 16 candidates positionally coincident with nearby $(d<250 \mathrm{pc})$ SFRs and 18 relatively bright $(V<14)$ "isolated" candidates. All our candidates have reported IR-excess from IRAS. Our aim was to determine whether the candidates are Herbig Ae/Be stars or background giants, and in the specific case of the candidates positionally coincident with SFRs, we wanted to further find out whether they are members of the SFR. We determined the spectral types of the sources by careful comparison with spectral templates, we measured their radial and projected rotational velocities, finally, we constrained their distances employing spectroscopic parallaxes based on the intrinsic colors of the established spectral type and luminosity class, and photometry from the literature.

From the 34 Herbig Ae/Be candidates studied, 26 objects exhibit $\mathrm{H} \alpha$ in emission ( $\sim 80 \%$, see Fig. 3 and Table 3$)$. From these 26 objects, 14 are dwarfs and subgiants (luminosity classes V and IV), 10 are giants and super giants (luminosity classes III, II, and I), and 2 are unclassified extreme emission line objects. From the 8 objects without $\mathrm{H} \alpha$ emission, 7 are giants and one (AS 321) is a main-sequence A-type star.

Among the 14 emission line dwarfs and subgiants, 13 objects are confirmed Herbig Ae/Be stars and one is a CTTS. In

11 A recently acquired high-resolution optical spectrum of AS 321 with UVES at the VLT (van den Ancker et al., in prep.) confirms the absence of $\mathrm{H} \alpha$ emission in the optical spectrum of AS 321. addition to these 13 confirmed Herbig Ae/Be stars, 5 additional sources might be Herbig Ae/Be stars: 3 emission-line early type luminosity class III giants, and 2 extreme emission line objects. However, our data have not allowed us to firmly establish whether these 5 sources are truly Herbig Ae/Be stars.

Two confirmed Herbig Ae/Be stars (CD-38 4380, HD 145718) and the CTTS (Hen 3-1145) are at distances closer than $250 \mathrm{pc}$. These sources are likely members of nearby SFRs. One emission line giant star (Hen 3-416) is closer than $300 \mathrm{pc}$. If this source is a young star, it may be associated with Sco OB 2-5. These 4 sources are likely to be nearby young stars and are interesting for follow-up observations at high-angular resolution. The rest of our confirmed Herbig Ae/Be stars (11 sources) are at distances greater than $700 \mathrm{pc}$. From this subsample, 7 stars (Hen 2-80, Hen 3-1121 N\&S, HD 313571, MWC 953, WRAY 15-1435, and Th 17-35) are inside or close (separation $<5^{\prime}$ ) to regions with extended infrared (IR) continuum emission at $8 \mu \mathrm{m}$ and have astronomical sources characteristic of SFRs in their 20' vicinity. These 7 sources are likely to be members of distant SFRs. Such regions are attractive for future studies of their stellar content. Two confirmed Herbig Ae/Be stars at $d>700$ pc, MWC 878 and WRAY 15-1372, may be truly "isolated" sources.

From our 34 Herbig Ae/Be candidates we found that $~ 50 \%$ (15 of 34) turned out to be background giant stars and not young stars. They show us that high-resolution optical spectroscopy is an important tool for distinguishing young stars (in particular Herbig Be stars) from post-main sequence stars in samples taken from catalogs based on low-resolution spectroscopy. A systematic study of large samples of candidates to Herbig Ae/Be stars employing high-spectral resolution spectroscopy is fundamental for firmly establishing their genuine young nature.

Acknowledgements. M.A. and A.C. acknowledge support from a Swiss National Science Foundation grant (PP002-110504). A.C. would like to thank C. Baldovin-Saavedra, F. Fontani, and A. Taylor for comments to the manuscript, J. Hernandez for discussions about the spectral classification of Herbig $\mathrm{Ae} / \mathrm{Be}$ stars, M. Chavez for providing a DVD with the UVBLUE/BLUERED spectral library, and A. Mueller for kindly making a set of high-resolution Kurucz synthetic spectra of low-mass stars available. We acknowledge the ESO and MPIA staff for carrying out the observations. This research made use of Aladin, the SIMBAD database and the VizieR service operated at the CDS, Strasbourg, France. This work is based in part on observations made with the Spitzer Space Telescope, which is operated by the Jet Propulsion Laboratory, California Institute of Technology under a contract with NASA.

\section{References}

Allen, D. A., \& Swings, J. P. 1976, A\&A, 47, 293

Bagnulo, S., Jehin, E., Ledoux, C., et al. 2003, Messenger, 114, 10

Bertone, E., Buzzoni, A., Chávez, M., \& Rodríguez-Merino, L. H. 2008, A\&A, 485,823

Böhm, T., \& Catala, C. 1993, A\&AS, 101, 629

Brown, J. M., Blake, G. A., Dullemond, C. P., et al. 2007, ApJ, 664, L107

Calvet, N., D'Alessio, P., Watson, D. M., et al. 2005, ApJ, 630, L185

Carmona, A. 2010, in proceedings of the conference Origin and Evolution of Planets 2008, Ascona, Switzerland, ed. L. Mayer, Earth, Moon, Planets, 106, 71

Dong, Y.-S., \& Hu, J.-Y. 1991, Acta Astrophys. Sinica, 11, 172

Finkenzeller, U., \& Mundt, R. 1984, A\&AS, 55, 109

Fontani, F., Beltrán, M. T., Brand, J., et al. 2005, A\&A, 432, 921

Fukagawa, M., Hayashi, M., Tamura, M., et al. 2004, ApJ, 605, L53

Ginestet, N., Carquillat, J. M., Jaschek, M., et al. 1992, Atlas de spectres stel-

laires de standards de classification MK, binaires spectroscopiques, étoiles particulières. Observatoire Midi-Pyrénées, Toulouse CRDP

Grady, C. A., Woodgate, B. E., Bowers, C. W., et al. 2005, ApJ, 630, 958

Gregorio-Hetem, J., \& Hetem, A. 2002, MNRAS, 336, 197

Guimarães, M. M., Alencar, S. H. P., Corradi, W. J. B., \& Vieira, S. L. A. 2006, A\&A, 457, 581

Hartmann, L. 1999, New Astron. Rev., 43, 1 
Herbig, G. H. 1960, ApJS, 4, 337

Hilton, J., \& Lahulla, J. F. 1995, A\&AS, 113, 325

Kaufer, A., Stahl, O., Tubbesing, S., et al. 1999, The Messenger 95, 8

Lang, K. R. 1991, Astrophysical Data: Planets and Stars (Springer)

Lanz, T., \& Hubeny, I. 2007, ApJS, 169, 83

Mannings, V., \& Sargent, A. I. 1997, ApJ, 490, 792

Merín, B., Jørgensen, J., Spezzi, L., et al. 2008, ApJS, 177, 551

Mikami, T., \& Heck, A. 1982, PASJ, 34, 529

Miroshnichenko, A. S., Fremat, Y., Houziaux, L., et al. 1998, A\&AS, 131, 469

Miroshnichenko, A. S., Levato, H., Bjorkman, K. S., \& Grosso, M. 2001, A\&A, 371,600

Miroshnichenko, A. S., Bjorkman, K. S., Grosso, M., et al. 2005, A\&A, 436, 653

Montes, D., Ramsey, L. W., \& Welty, A. D. 1999, ApJS, 123, 283

Mottram, J. C., Vink, J. S., Oudmaijer, R. D., \& Patel, M. 2007, MNRAS, 377, 1363

Morgan, W. W., Keenan, P. C., \& Kellman, E. 1943, Chicago, Ill. (The University of Chicago press)

Muzerolle, J., D’Alessio, P., Calvet, N., \& Hartmann, L. 2004, ApJ, 617, 406

Najita, J. R., Carr, J. S., Glassgold, A. E., \& Valenti, J. A. 2007, in Protostars and Planets V, 507

Palla, F., Brand, J., Comoretto, G., Felli, M., \& Cesaroni, R. 1991, A\&A, 246, 249

Phillips, C. J., Norris, R. P., Ellingsen, S. P., \& McCulloch, P. M. 1998, MNRAS, 300,1131
Pontefract, M., Drew, J. E., Harries, T. J., \& Oudmaijer, R. D. 2000, MNRAS, 319, L19

Preibisch, T., \& Mamajek, E. 2008, Handbook of Star Forming Regions, Vol. II, 235

Reipurth, B., Pedrosa, A., \& Lago, M. T. V. T. 1996, A\&AS, 120, 229

Sarkar, G., Parthasarathy, M., \& Reddy, B. E. 2005, A\&A, 431, 1007

Schmidt-Kaler, Th. 1982, in Landolt/Bornstein, New Series Group VI, 2

Schönberner, D., \& Drilling, J. S. 1984, ApJ, 278, 70

Schultz, G. V., \& Wiemer, W. 1975, A\&A, 43, 133

Semenov, D., Pavlyuchenkov, Y., Schreyer, K., et al. 2005, ApJ, 621, 853

Soubiran, C., Katz, D., \& Cayrel, R. 1998, VizieR Online Data Catalog, 413, 30221

Suárez, O., García-Lario, P., Manchado, A., et al. 2006, A\&A, 458, 173

Szczerba, R., Siódmiak, N., Stasińska, G., \& Borkowski, J. 2007, A\&A, 469, 799

Thé, P. S., de Winter, D., \& Perez, M. R. 1994, A\&AS, 104, 315

van den Bergh, S. 1966, AJ, 71, 990

Vieira, S. L. A., Corradi, W. J. B., Alencar, S. H. P., et al. 2003, AJ, 126, 2971

Vink, J. S., Drew, J. E., Harries, T. J., \& Oudmaijer, R. D. 2002, MNRAS, 337, 356

Vink, J. S., Harries, T. J., \& Drew, J. E. 2005, A\&A, 430, 213

Waters, L. B. F. M., \& Waelkens, C. 1998, ARA\&A, 36, 233

de Zeeuw, P. T., Hoogerwerf, R., de Bruijne, J. H. J., Brown, A. G. A., \& Blaauw, A. $1999, \mathrm{AJ}, 117,354$

Pages 15 to 22 are available in the electronic edition of the journal at http://www. aanda.org 


\section{Appendix 1. Comments on the spectral classification of individual sources}

\section{AS 231}

In AS 231 we observe $\mathrm{H} \gamma, \mathrm{H} \beta, \mathrm{H} \alpha$, HeI, Ca I (3933 $)$ ), Fe II (4233, $4352 \AA)$, and Ti I (4550, $4583 \AA$ ) P-Cygni profiles. Given that He II absorption lines are absent in the spectrum, the presence of a few He I lines in absorption indicates that AS 231 is a B star. As the Mg II line at $4481 \AA$ is much stronger than the He I line at $4471 \AA$ and the He I line at $4009 \AA$ is absent from the spectrum, AS 231 should have a spectral type later than B8. Since Si II lines are observed at 3854, 3856, 3863, 4128, and $4131 \AA$ A AS 231 has luminosity class I. The absence of the He I line at $4144 \AA$, the lack of strong C II line at $4267 \AA$ and the presence of Fe II in absorption at 4173, 4179, and $4417 \AA$, rule out the spectral type B8, and indicate that AS 231 should have a spectral type B9. The presence of a relatively strong Fe II lines at 4179,4233 , and $4352 \AA$ further indicates that the luminosity class is Ia. We conclude that AS 231 has a B9Iae spectral type.

\section{AS 321}

AS 321 does not show hydrogen emission lines in our spectrum. Hydrogen is observed in absorption and the profiles are broad. A strong and broad Ca II $\mathrm{K}$ line in absorption is present. These characteristics indicate that AS 321 is an A-type star. The width and the strength of the CaII $\mathrm{K}$ line and the hydrogen lines indicate that AS 321 is a mid-A star. The width of the hydrogen lines, the width and shape of the Fe absorption lines, the broad Ca I line at $4227 \AA$ and the absence of lines such as the Ti II lines at 3901 and $3913 \AA$, rule out the luminosity classes I and II. The profile's shape of the Fe lines can be reasonably matched with the spectrum of A7III and A8III stars once rotational broadening is taken into account. However, since the hydrogen lines profiles obtained are narrower than those of our FEROS spectrum, we deduce that AS 321 should be of luminosity class V. The spectral template that best fits the observations of AS 321 is the one of an $\mathrm{A} 5 \mathrm{~V}$ star. Therefore, we conclude that the spectral type of AS 321 is A5V.

\section{CD-38 4380}

CD-38 4380 exhibits $\mathrm{H} \alpha$ in emission. Its spectrum does not exhibit He I or He II absorption lines. Several weak absorption lines are observed in between the Balmer lines. This combined with the strength of the Ca II K line at $3933 \AA$ indicates that CD-38 4380 must be later than A9. The lack of a strong G-band feature at $4300 \AA$ shows that CD-38 4380 is earlier than G-type. Therefore, CD-38 4380 is most likely a F-type star. The width of the Balmer lines at 3889 and $3970 \AA$ further indicates that CD-38 4380 should have a spectral type later than F3. The strength of the $\mathrm{CaI}$ line at $4227 \AA$, the Fe I lines at 4005 and $4144 \AA$, and the SrII line at $4078 \AA$ line indicates that CD-38 4380 should be of spectral type earlier than F6. To determine the luminosity class we analyzed the region around the Ca I line at $4227 \AA$. The strengths the Sr II line at $4216 \AA$, the Ca I line at $4227 \AA$, the Fe II line at $4247 \AA$, and other absorption lines observed in the region are too weak to be compatible with the luminosity classes I and II. Templates of F2 to F4 class III giants are compatible with the general shape of the observed spectrum. However, the best match is provided by a rotationally broadened $\left(v \sin (i) 45 \mathrm{~km} \mathrm{~s}^{-1}\right) \mathrm{F} 4 \mathrm{~V}$ template. Thus, we conclude that CD-38 4380 has a F4Ve spectral type. Previous studies based on low resolution spectra suggested spectral types F3V (Vieira et al. 2003, PDS 277) and F2Ie (Suaréz et al. 2006). Our data rule out the F2 supergiant spectrum. F3V is compatible with the observed data, but our high resolution spectrum is better described by a F4Ve spectrum.

\section{HD 145718}

HD 145718 displays an inverse P Cygni $\mathrm{H} \alpha$ profile. Other Balmer lines are observed in absorption, but their profiles are not symmetric. The blue part displays a shoulder consistent with filling with an emission component. The spectrum lacks of He I lines and a broad Ca II K line at $3933 \AA$ is observed. The strength the Ca II K line shows that HD 145718 is an A-type star. Its width indicates that HD 145718 has a spectral type later than A3 but earlier than A7. The strength of the Mg II line at $4481 \AA$ constrains the spectral type to be earlier than A6. The strength of the Ca I line at $4227 \AA$ constrains the spectral type to be later than A4. Comparison with the spectral templates shows that the spectral type that best match the observed spectrum is A5. The weak strength of the Fe II - Ti II lines at $4173 \AA$, the Y II Fe II lines at $4179 \AA$, the Ti II lines at 3901 and $3913 \AA$ rules out the luminosity classes I and II. Analyzing the region between the $\mathrm{H} \gamma$ line and the Ti II line at $4583 \AA$, we found that the width of the weak absorption features visible in the spectrum are much better matched by a dwarf star (luminosity class V) than with a rotationally broadened giant (luminosity class III). Therefore we conclude that HD 145718 is a dwarf star and has a spectral type A5Ve. HD 145718 was studied by Guimaraes et al. (2006) who found a $T_{\text {eff }}=7500 \pm 200 \mathrm{~K}$, corresponding to a somewhat later spectral type than the A5Ve found by us. However, we note, as previously mentioned, that the strength of the Mg II line at $4481 \AA$ indicates that the spectral type is earlier than A6. Comparison of the $100 \AA$ region around the $\mathrm{Mg}$ II line at $4481 \AA$ with BLUERED synthetic spectra indicates that the $T_{\text {eff }}$ that best matches our HD 145718 spectrum is in the $8000-8500 \mathrm{~K}$ range (for $\log g$ ranging from 3.0 to 4.0). This further indicates that the spectral type of HD 145718 is A5Ve.

\section{HD $152291=$ MCW 1264}

HD 152291 displays $\mathrm{H} \alpha$ in emission. Its spectrum exhibits $\mathrm{He}$ I in absorption. Given that no He II absorption lines are observed, HD 152291 should have a spectral type later than B0.5. The presence of the He I line at $4121 \AA$ and a weak Mg II line at $4481 \AA$ indicates that HD 152291 is earlier than B5. The presence of a strong O II - C III blend at $4650 \AA$ shows that HD 152291 is earlier than B2. The presence of the O II lines at 4070, 4300 and $4415 \AA$ rules out the luminosity class V. The lack of the N II lines at 3995 and $4631 \AA$, and the presence of strong Si III triplet at $4553 \AA$ rule out the luminosity class I-II. Therefore, we conclude that HD 152291 has a spectral type B1IIIe.

\section{HD 305773}

HD 305773 does not show hydrogen emission lines. He I absorption lines at 4026, 4144, 4471, 5016, 5876, 6678, and $7066 \AA$ are observed. Since He II lines are absent, HD 305773 should have a spectral type B. The presence of the He I line at 4009, 4121, and $4388 \AA$ indicates that the spectral type is earlier than B5. The detection of the N II line at $3995 \AA$, the O II lines at 4070 and 
$4976 \AA$, the Si III lines at 4553,4568 , and $4575 \AA$ indicates that the luminosity class of HD 305773 is I. The lack of the Si II lines at 4128 and $4131 \AA$ shows that HD 305773 is earlier than B3. The presence of the Si IV lines at 4089 and $4016 \AA$ indicates that HD 305773 should be of spectral types B0-B1. The presence of a rich spectra of $\mathrm{O}$ II lines and the strength of the $\mathrm{Mg}$ II line at $4481 \AA$ constrain the spectral range to be B1 or later. Therefore, HD 305773 should have a spectral type B1. The detection of the $\mathrm{C}$ II line at $4267 \AA$ indicates that the luminosity class is $\mathrm{Ib}$. In summary, HD 305773 has a B1Ib spectral type.

\section{HD $313571=$ MWC 595}

The spectrum of HD 313571 shows $\mathrm{H} \alpha, \mathrm{H} \beta, \mathrm{H} \gamma$, and $\mathrm{H} \delta$ in emission. Double peaked hydrogen Paschen lines are observed in emission at $8000 \AA$. The Ca II triplet at $8500 \AA$ is observed in emission superposed to the hydrogen Paschen lines. He I is observed in absorption (e.g., at 4026, 4144, 4121, 4386, 4471, and $4713 \AA$ ) and in emission (e.g., 4922, 5016, 5876, 6678, and $7066 \AA$ ). Since He II lines are not present in the spectrum, HD 313571 is a B-type star. The lack of the He II line at $4686 \AA$ shows that HD 313571 has a spectral type later than B0.5. The Mg II line at $4481 \AA$ is present and it is weaker than the He I line at $4471 \AA$, thus HD 313571 should have a spectral type earlier than B6. The strength of the He I line at $4009 \AA$ indicates that HD 313571 should have a spectral type B3 or earlier. The absence of the N II line at $3995 \AA$, the Si II lines at 4128 and $4131 \AA$, the Si III lines at 4553,4568 , and $4575 \AA$, the Si IV line at 4089 and $4116 \AA$, and O II lines in the regions at 4000, 4300, and $4400 \AA$, indicates that HD 313571 is not a supergiant star (luminosity classes I and II). The lack of O II absorption at 4415 and $4417 \AA$ rules out the spectral type B1 III. The strength of the $\mathrm{Mg}$ II line at $4481 \AA$ suggests a spectral type later than B1. The lack of Si III lines at 4553,4568 , and $4575 \AA$ rules out the spectral type B2III. The absence of the Si II lines at 4128 and $4131 \AA$ indicates a spectral type later than B2V. The strength of the $\mathrm{HeI}$ line at $4009 \AA$ with respect to the $\mathrm{He}$ I line at $4026 \AA$ and the presence of a the $\mathrm{C}$ II line at $4267 \AA$ rule out the spectral types B4III-V. Comparison with spectral templates shows that the spectrum that better matches the relatively intensity of the $\mathrm{He}$ I lines is the spectral type B3V. We conclude that HD 313571 has a B3Ve spectral type.

\section{$H D 320156=$ Hen $3-1444$}

HD 320156 displays $\mathrm{H} \alpha$ and $\mathrm{H} \beta$ in emission. Inverse $\mathrm{P}$ Cygni He II profiles are observed. The He II line at $4686 \AA$ is absent. A rich spectrum of He I lines is observed. The He I lines are narrow, typically of $F W H M \sim 45 \mathrm{~km} \mathrm{~s}^{-1}$, some of them display inverse $\mathrm{P}$ Cygni profiles, and in some cases double peaked profiles. The Mg II line at $4481 \AA$ is present and has a strength similar to the He I line at $4471 \AA$. This ensemble of characteristics suggests that HD 320156 is an early B-type star. The absence of the He II line at $4686 \AA$ indicates that HD 320156 has a spectral type B1 or later. An He I line at $4009 \AA$ of similar strength than the He I line at $4026 \AA$ narrows down the spectral types to B2 to B4. The spectrum displays C II lines at 3919 and $3921 \AA$, N II at $3995 \AA$, Si II at 4128 and $4131 \AA$, and Si III at 4553, 4568, and $4575 \AA$. This, combined with the narrow $\mathrm{H}$ lines observed in absorption, rules out the luminosity classes II, III, and V, and shows that HD 320156 is a supergiant star of luminosity class I. The presence of the mentioned Si II and Si III lines, and the lack of strong O II lines at 4070, 4076, 4346, and $4649 \AA$ indicate that its spectral type should be B3 to B4. The similar strength of the He I line at $4121 \AA$, and the Si II lines at 4128 and $4131 \AA$ indicates a luminosity class Ib and suggests a spectral type B4. We conclude that HD 320156 has a spectral type B4Ibe. Finally, we note that Schönberner \& Drilling (1984) suggested that HD 320156 (=LSS 4300) is a close binary system consisting of a helium supergiant of $\sim 1 M_{\odot}$ and a less luminous secondary. Our FEROS spectrum is compatible with this hypothesis.

\section{HD $323154=$ MWC 877}

HD 323154 displays a flat spectrum characterized by the presence of He I in absorption at 3820, 4009, 4121, and $4713 \AA$. He I is observed in emission starting with the line at $4922 \AA$. No He II lines are present in the spectrum. Hydrogen lines are observed in emission starting with the $\mathrm{H} \delta$ line at $4102 \AA$. The $\mathrm{H} \alpha$ line exhibits a double peaked profile. Broad hydrogen Paschen lines are observed in emission starting at $8360 \AA$. The lack of He II lines and the presence of He I lines indicate that HD 323154 is a B star. The Mg II line at $4481 \AA$ has an absorption depth slightly weaker than the He I line at $4471 \AA$ A. This indicates that HD 323154 has a spectral type earlier than B6 (given that the He I profile is contaminated by emission, we can only use the $\mathrm{Mg}$ II/He I ratio to set an upper spectral type limit). The presence of N II absorption at $3995 \AA$ rules out the luminosity class V and IV for HD 323154, and indicates that HD 323154 has a spectral type earlier than B5. The weak C II line at $4267 \AA$ and the lack of O II lines at 4070, 4076, 4346, 4349, 4415, and $4417 \AA$ rule out the spectral types B1I-III and B2I-III. The strength of the C II line is weaker than observed in B3I-III stars, thus HD 323154 should have a spectral type B4. A strength of the Mg II line similar to that of the He I $4471 \AA$ A line and the strength of Si II lines at 4128 and $4131 \AA$ rule out the luminosity class I. The presence of Si III absorption at $4553 \AA$ and the N II line at $4631 \AA$ are inconsistent with the luminosity class III. Therefore, the luminosity class of HD 323154 should be II. We conclude that HD 323154 has a spectral type B4IIe.

\section{Hen 2-80}

The spectrum of Hen 2-80 is flat and it exhibits only a few absorption lines. The presence of weak He I lines at 4026, 4144, and $4471 \AA$ suggests that Hen $2-80$ has a spectral type earlier than B8. Since the Mg II line at $4481 \AA$ is just slightly weaker than the He I line at $4471 \AA$, Hen 2-80 should have a spectral type B5 or B6. The absence of the He I $4009 \AA$ and the presence of the He I at $4026 \AA$ suggest that Hen 2-80 has a spectral type B6 or later. The lack of the Si II triplet at $3855 \AA$ A rules out the luminosity classes I and II. Given that the Mg II line width is much narrower than the He I line at $4471 \AA$, Hen 2-80 is not of luminosity class III. We conclude that Hen 2-80 has a spectral type B6Ve.

\section{Hen 3-416}

Hen 3-416 exhibits P-Cyni profiles in the Balmer lines. Strong $\mathrm{H} \alpha$ in emission is observed. Ca II emission at 8498, 8542, and $8662 \AA$ is observed as well. He I lines in absorption are not present in the spectrum. However, the He I lines at 4922 and 
$5016 \AA$ display P-Cygni profiles. The lack of He I absorption points to a star of spectral type A or later. The lack of the Ca I line at $4227 \AA$ indicates that Hen 3-1416 should be of spectral type earlier than A3. A narrow and relatively strong $\mathrm{Ca}$ II $\mathrm{K}$ line at $3933 \AA$ and the strength of the Mg II line at $4481 \AA$ are consistent with a spectral type earlier than A2. The very narrow Balmer lines indicate that Hen 3-416 is not a dwarf (i.e. not of luminosity class V). The lack of multiple absorption lines in the region of around the Mg II line at $4481 \AA$ and the Ca I line at $4227 \AA$ rules out the luminosity classes I and II. The strength of the Fe II line at $4233 \AA$ shows that the spectrum is later than A0. The lack of Ca I absorption at $4227 \AA$ rules out the spectral type A2III. This ensemble of diagnostics suggests that Hen 3-416 has a spectral type A1III. We conclude that Hen 3-416 has an A1IIIe spectral type.

\section{Hen $3-823=C D-594412$}

Hen 3-823 displays broad $\mathrm{H} \alpha$ and $\mathrm{Ca}$ II in emission. Its spectrum is flat and exhibits several lines of He I. Since He II absorption is not observed, in particular the line at $4686 \AA$, Hen 3-823 should have a spectral type B later than B0.5. The presence of the He I lines at 4009, 4121, 4026, 4388, and $4471 \AA$ and a Mg II line at $4481 \AA$ weaker than the He I line at $4471 \AA$ indicate that Hen 3-823 should have a spectral type earlier than B5. The strength of the Mg II and the He I line at $4009 \AA$ suggests that Hen 3-823 should have a spectral type B2 or later. The strength of the He I lines at $4121 \AA$ and $4388 \AA$ shows that Hen 3-823 should have a spectral earlier than B4. The lack of the Si III lines at 4553, 4568, and $4575 \AA$, and O II lines at 4070, 4349 , and $4415 \AA$ rules out the luminosity classes I and II. Broad He I absorption profiles and the absence of He I at $3927 \AA$ rule out the luminosity class III and indicate that Hen $3-823$ is a dwarf. The strength of the He I lines is best matched by a B3 star. We conclude Hen 3-823 has a spectral type B3Ve.

\section{Hen 3-1121N}

Hen 3-1121N exhibits a weak $(E W=-0.3 \AA)$ narrow $(F W H M=$ $35 \mathrm{~km} \mathrm{~s}^{-1}$ ) $\mathrm{H} \alpha$ line in emission observed inside a broad $\mathrm{H} \alpha$ line in absorption $\left(F W H M=460 \mathrm{~km} \mathrm{~s}^{-1}\right)$. The spectrum is rich in He I absorption lines. Since He II at $4686 \AA$, C III at 4647 and $4651 \AA, C$ II at $4267 \AA$, and Si IV at 4089 lines are observed in absorption, Hen $3-1121 \mathrm{~N}$ should have a spectral type earlier than B1. The lack of the He II line at $4542 \AA$ rules out the late $\mathrm{O}$ spectral types and indicates that Hen $3-1121 \mathrm{~N}$ has an early B spectral type. Presence of the Si IV line at $4089 \AA$, the lack of the Si IV line at $4116 \AA$ and the lack of strong Si III lines at 4553,4568 , and $4575 \AA$ suggest that Hen $3-1121 \mathrm{~N}$ is of spectral type B0.5. The lack of O II absorption lines at 4317, 4320, $4346,4349,4367,4415$, and $4417 \AA$ rules out the luminosity classes I-III. We conclude that Hen $3-1121 \mathrm{~N}$ has a spectral type B0.5Ve.

\section{Hen 3-1121S}

Hen 3-1121S displays a broad $\mathrm{H} \alpha$ line, a very weak emission component $(E W=-0.05 \AA)$ is observed inside the absorption line. The spectrum exhibits a rich spectra of He I lines. He II is observed in absorption at $4686 \AA$ and no other He II lines are observed. C III absorption is observed at 4647 and $4651 \AA$. These spectral characteristics indicate that Hen 3-1121S has a spectral type B earlier than B1. The Si IV line at $4089 \AA$ is stronger than the Si III line at $4552 \AA$ indicating that the spectral type is B0. The presence of the He II line at $4686 \AA$ combined with the width and shape of the hydrogen Balmer lines and the absence of O II lines at 4317, 4320, 4346, 4349, and $4367 \AA$, and the N II line at $3995 \AA$ indicate that the star is not of luminosity class I or II. The strength of the He I 4009 relative to the strength of the He I $4026 \AA$ line indicates that Hen $3-1121 \mathrm{~S}$ is of luminosity class V. We conclude that Hen $3-1121 \mathrm{~S}$ has a spectral type B0Ve.

\section{Hen 3-1145}

Hen 3-1145 displays a spectrum rich in emission lines. We observe in emission hydrogen Balmer lines $(\mathrm{H} \alpha, \mathrm{H} \beta, \mathrm{H} \gamma, \mathrm{H} \delta, \mathrm{H} v$, $\mathrm{H} \xi$ ), He I at 4026, 4471, and $5876 \AA$, He II at $4686 \AA$, Ca II at $3933 \AA$ and the Ca II triplet at $8500 \AA$. Li is observed in absorption at $6708 \AA(E W=0.5 \AA)$. The spectrum is also rich in narrow absorption lines, thus Hen 3-1145 should have a late spectral type. The strength and width of the Ca I line at $4227 \AA$ shows that Hen 3-1145 has a spectral type $\mathrm{K}$ or later (note that the $\mathrm{Ca}$ I line is likely to be contaminated by emission; thus, the real $\mathrm{Ca}$ I strength is intrinsically larger than observed). The presence of weak molecular bands at $7000 \AA$ indicates that Hen 3-1145 has a spectral type later than K7 but earlier than M2 (otherwise the molecular bands will be much stronger). The strength of the Na I D lines at $5890 \AA$ and the molecular bands at $7000 \AA$ indicate that Hen 3-1145 has a spectral type M0 to M1. The smallest residuals to the molecular bands at $7000 \AA$ are given by the spectral type M1. The absence of the absorption doublet at $5860 \AA$ further indicates that the spectral type should be M1. The broad width of $\mathrm{Na}$ I D lines rules out the luminosity class III (Montes et al. 1999). Comparison of the Na I D lines with synthetic spectra shows that the line is slightly narrower than observed in the luminosity class $\mathrm{V}$ thereby indicating that the luminosity class of Hen 3-1145 is IV. We conclude that Hen 3-1145 has a spectral type M1IVe. Fitting of the spectra with high-resolution synthetic models of a M1 star $\left(T_{\text {eff }}=3720\right)$ shows that the $\log (g)$ that better describes the spectra is 4.0 (we used these values for deriving the absolute magnitude of Hen 3-1145 and constrain its distance, see Sect. 3.2).

\section{Hen $3-1347=B D-184436$}

The spectrum of Hen 3-1347 exhibits $\mathrm{H} \delta, \mathrm{H} \gamma, \mathrm{H} \beta, \mathrm{H} \alpha$ emission lines. He II absorption is not present. He I in absorption is observed at 4009, 4121, 4713, 4922, 5116, 5876, and $6678 \AA$. The absence of He II lines and the presence of He I absorption lines indicate that Hen 3-1347 is a B star. The presence of the He I at $3965 \AA$ de-blended from a narrow $\mathrm{H} \epsilon$ line, the narrow Balmer line wings, combined with the presence of $\mathrm{N}$ II absorption at $3995 \AA$, Si II at 4128 and $4131 \AA$, C II at $4267 \AA$, and Si III at 4553, 4568, and $4575 \AA$, indicate that Hen 3-1347 has a luminosity class I. A Mg II line at $4481 \AA$ slightly stronger that the He I line at $4471 \AA$ suggest a spectral type B5 or later. The presence of the He I line at $4009 \AA$ rules out the spectral types later than B6. The presence of Si III lines at 4553, 4568, and $4575 \AA$, and the He I line at $4121 \AA$ suggests a luminosity class Ib. The presence of the N II line at $3995 \AA$ and the mentioned Si III lines 
are better described by the spectral type B5 than B6. We conclude that Hen 3-1347 has a B5Ibe spectral type.

\section{Hen 3-1428}

Hen 3-1428 exhibits He I in absorption. Since He II lines are absent from the spectrum, Hen 3-1428 should have a spectral type B. Hydrogen Balmer and Paschen lines are observed in emission displaying P-Cygni profiles. He I lines at 4471, 4713, 5016, and $5876 \AA$ exhibit P-Cygni profiles as well. No Ca II emission is observed at $8000 \AA$. O I forbidden emission is observed at 5577, 6300, and $6364 \AA$. N II emission is observed at 6548 and $6584 \AA$ showing a P-Cyni profile (the line at $5755 \AA$ is not present). The Mg II line at $4481 \AA$ is present and is weaker than the He I line at $4471 \AA$ (note, however that this He I line has PCygni profile) and is relatively broad ( $F W H M=$ $78 \mathrm{~km} \mathrm{~s}^{-1}$ ). The weak Mg II line at 4481 and the presence of a relatively strong He I line at $4009 \AA$ indicate that Hen 31428 should be of spectral type earlier than B3. The presence of C III lines at 4647 and $4651 \AA$ indicates that Hen 3-1428 should have a spectral type B2 or earlier. Since in the spectrum are simultaneously present the He I line at $4009 \AA$, and the O II lines at 4317 and $4320 \AA$, the spectral type B0 is ruled out, thus the spectral type should be B1 or B2. The presence of the N II line at $4631 \AA$, and the O II lines at 4639, 4642, and $4650 \AA$ indicates that Hen 3-1428 is of luminosity class I or II. As the N II at $3995 \AA$ line is stronger than the C II lines at $3919 \AA$, luminosity class of the spectrum should be class I. The strength of the O I lines at 4415 and $4417 \AA$ suggests a spectral type is B1I. Finally, the luminosity class Ia describes better the strength of the N II 3995/He I 4009 line ratio and the relative strength of the lines observed in the region of the N II line at $5680 \AA$. We conclude that Hen 3-1428 has a B1Iae spectral type.

\section{MWC 314}

MWC 314 has a spectrum rich in emission lines of hydrogen (Balmer and Paschen series), He I, He II, Ti II, Ca II, Si II, Mg II, and Fe II. Although the spectrum is strongly influenced by the presence of emission lines several absorption lines are observed: $\mathrm{N}$ II at $3995 \AA$, He I at 4009, 4026, 4121, 4388, and $5047 \AA$, S II at 5453, 5473, and $5640 \AA$, Al III at 5696 and $5722 \AA$, and $\mathrm{Ne}$ I at 6143,6364 at $6462 \AA$. The absence of He II in absorption and the detection of He I lines indicate that MWC 314 is a B-type star. In particular the presence of the He I lines at 4009 and $4026 \AA$ indicates that the spectral type of MWC 314 should be early than B7 and later than B0. The detection of the $\mathrm{S}$ II lines rules out the luminosity classes $\mathrm{V}$ and III. The observation of the $\mathrm{N}$ II, $\mathrm{Al}$ III, and Ne I lines indicates that the luminosity class is I. The presence of the He I line at $4009 \AA$, the absence of the Si IV lines at 4089 and $4116 \AA$, and the $\mathrm{C}$ II line at $4267 \AA$ indicate that MWC 314 should have a spectral type later than B1. The strength of the He I lines at 4009 and $5046 \AA$, and the absence of the Si II lines at 4128 and $4131 \AA$ indicate that MWC 314 has a spectral type B3 or earlier. The absence of the Si III lines at 4553, 4568, and $4575 \AA$ indicates that MWC 314 should have a spectral type later than B4 if it has luminosity class Ia and later than B3 if it has of luminosity class Ib. To match the Si II and Si III requirements, MWC 314 should have a spectral type B3 and luminosity class Ib. We conclude that MWC 314 has a spectral type B3Ib. We note that Miroshnichenko et al. (1998) suggest a spectral type B0 for MWC 314. This spectral type is inconsistent with our FEROS data, in particular because of the presence of the He I line at $4009 \AA$, and the non detection of the Si IV lines at 4089 and $4116 \AA$, the C III lines at 4068, 4070, 4647, and $4651 \AA$, the O II line at $4076 \AA$, and the Si III lines at 4553, 4568, and $4575 \AA$.

\section{MWC $593=C D-2413510$}

MWC 593 exhibits hydrogen Balmer and Paschen emission. The Paschen lines are broad $\left(F W H M \sim 500 \mathrm{~km} \mathrm{~s}^{-1}\right)$ and double peaked. No He II is observed in absorption. He I is present, in particular the lines at 4009,4121 , and $4388 \AA$. The Mg II line at $4481 \AA$ is present, but it is very weak in contrast to the He I line at $4471 \AA$. These characteristics indicate that MWC 593 is a B star of spectral types between B1 and B5. The lack of C II at 3919 and $3921 \AA, N_{\text {II }}$ at $3995 \AA$, He I at $3927 \AA$, Si II at 4128 and $4131 \AA$, and Si III at 4553, 4568, and $4575 \AA$ shows that MWC 593 is not a giant (luminosity classes I, II, or III) and indicates that MWC 593 should have a spectral type B3 or B4. Comparison with spectral templates shows that the spectra that better match the relative intensity of the He I lines and the Mg II line is the spectral type B4. We conclude that MWC 593 has a spectral type B4Ve.

\section{MWC $878=C D-3811837=$ Hen $3-1398$}

MWC 878 displays a flat spectrum rich in emission lines. We observe in emission hydrogen Balmer lines $(\mathrm{H} \delta, \mathrm{H} \beta, \mathrm{H} \alpha)$, double peaked broad $\left(F W H M \sim 390 \mathrm{~km} \mathrm{~s}^{-1}\right)$ hydrogen Paschen lines starting at $8370 \AA$, double peaked broad $\left(F W H M \sim 360 \mathrm{~km} \mathrm{~s}^{-1}\right)$ Ca II lines at 8498, 8542, and $8662 \AA$, He I lines at 4009, 4471, 4713, 5876, 6678, 7066, and $7281 \AA$, He II lines at 4542, $4787 \AA$, the O I line at $6300 \AA$, O III lines at 4959 and $5007 \AA$, $\mathrm{N}$ II lines at 5755,6548 , and $6584 \AA$, the S III line at $6312 \AA$, and Ar III lines at 7136 and $7753 \AA$. Very few absorption lines are visible, a narrow $\left(F W H M \sim 40 \mathrm{~km} \mathrm{~s}^{-1}\right) \mathrm{Ca}$ II line at $3933 \AA$ and the Na I D lines at $5890 \AA$. We did the spectral classification based on the region around the He I lines at 4009 and $4471 \AA$. The presence of $\mathrm{He}$ I at $4009 \AA$ in absorption indicates that the star is of spectral type earlier than B6. The absence of a Mg II line at $4481 \AA$ indicates that MWC 878 has a spectral type earlier than B2 and indicates that MWC 878 is very likely not a giant or supergiant (luminosity classes III, II, and I). Since the Si IV line at $4089 \AA$, the Si III lines at 4553, 4568, and $4575 \AA$, the C II line at $4267 \AA$ are absent from the spectrum, MWC 878 should have a spectral type later than B0.5. We conclude that MWC 878 has a B1Ve spectral type. For this star, Miroshnichenko et al. (2001) derived a spectral type of O9/B0, these spectral types are incompatible with our FEROS spectrum.

\section{MWC 930}

MWC 930 has a spectrum rich in emission lines. Hydrogen Balmer and Paschen, He I, strong Ca II, S II, C III, N II, Fe II, and [Fe II] lines are observed displaying P-Cygni profiles. The Na I D lines at $5890 \AA$ are saturated and display a complex structure, double absorption and three emission peaks. The Mg II line at $4481 \AA$ is observed in absorption and it is relatively strong $(E W=1.4 \AA)$. Since the lines used for spectral classification are affected by emission components, the spectral classification is highly unreliable because these lines are likely to arise in an extended atmosphere or shell. The strong veiling also affects the 
spectral classification. We attempt here to constrain the spectral classification based on the absence of strong spectral features. Since the He II line at $4686 \AA$ is absent, MWC 930 should have a spectral type later than B0. The detection of the He I line at $5876 \AA$ implies that MWC 930 is a B-type star. The strong Mg II line at $4481 \AA$ indicates that MWC 930 should have a spectral type later than B5. The lack of absorption lines does not allow us to establish the luminosity class. We conclude MWC 930 should have a spectral type B5-B9.

\section{MWC 953}

MWC 953 exhibits $\mathrm{H} \delta$ to $\mathrm{H} \alpha$ in emission. Its spectrum shows $\mathrm{He} \mathrm{I}$ in absorption and no He II lines are observed. Since the He II line at $4686 \AA$ is absent, MWC 953 should have a spectral type later than B0.5. The absence of the Si IV line at $4089 \AA$ and the Si III line at $4452 \AA$ indicates that the spectral type of MWC 953 is later than B1. The Mg II at $4481 \AA$ line is present and is much weaker than the He I line at $4471 \AA$, thus MWC 953 should have a spectral earlier than B5. The lack of Si II lines at 4128 and $4131 \AA$, and O II lines at 4070, 4076, 4349, 4415, and $4417 \AA$ rules out the luminosity classes I and II for the spectral types B2 to B5. The presence of the C II line at $4267 \AA$ rules out the spectral types B3V, B4V and B5V. The strength of the Mg II line at $4481 \AA$ is not compatible with the spectral types B4III and B5III. The strength of the He I line at $4713 \AA$ and the $\mathrm{Mg}$ II line at $4481 \AA$ is not consistent with the spectral type B3III. The weak strength of Si III in absorption at 4553, 4568 , and $4568 \AA$ rules out the spectral type B2III. Therefore, we conclude that MWC 953 has a spectral type B2Ve.

\section{Th 17-35}

Th 17-35 exhibits $\mathrm{H} \alpha$ and $\mathrm{Ca}$ II in emission. Its spectrum is flat and besides Balmer lines with emission components, very few absorption lines are observed. No He II lines are observed. A He I line at $5976 \AA$ and a weak He I line at $4471 \AA$ are present. In consequence, Th 17-35 is a B-type star. The lack of strong He I lines in the $4000 \AA$ region indicates that Th $17-35$ is of spectral type later than B6. The Mg II line at $4481 \AA$ is weak but has a strength similar to that of the He I line at $4471 \AA$, suggesting that Th 17-35 should have a spectral type later than B7. The weak Mg II line shows that the spectral type should be earlier than B9. Thus, Th 17-35 should have a spectral type B8. The lack of a strong Mg II line at $4481 \AA$ rules out the luminosity classes I and II. The absence of the He I line at $4026 \AA$ rules out the luminosity class III. We conclude that Th 17-35 has a B8Ve spectral type. Vieira et al. (2003) suggested a B2V spectral type for this star. B2 stars display the strongest He I absorption lines of the B spectral class. Since we observe an almost flat spectrum, our data rule out the $\mathrm{B} 2 \mathrm{~V}$ type.

\section{Th 35-41}

Th 35-41 does not show $\mathrm{H} \alpha$ in emission. The general shape of the spectrum reveals a late-type star spectrum (i.e. spectral types K or M). The narrow shape of the Na I D lines at $5980 \AA$ indicates that Th $35-41$ is a giant star, and suggests a spectral type later than M0III. The lack of strong molecular bands further indicates that Th 35-41 is earlier than M5III. The strength of the absorption lines observed in the region close to the Na I D lines further constrains the spectral type to be earlier than M3III. The best fit to the regions around the $\mathrm{NaI} \mathrm{D}, \mathrm{H} \alpha$ and $\mathrm{Ca}$ II ( $\sim 8500 \AA$ ) lines (see Montes et al. 1999) was given by the spectral type M1III. Thus, we conclude that Th $35-41$ has a spectral type M1III.

\section{WRAY 15-488}

WRAY 15-488 exhibits $\mathrm{H} \alpha$ and $\mathrm{H} \delta$ P Cyni profiles with a strong absorption component and a weak emission component. This suggests the presence of a wind. He I lines are not present in the spectra, thus ruling out the spectral types O and B. Since the spectrum displays a relatively large amount of broad absorption lines, WRAY 15-488 should have a spectral type later than A. The strength of the Fe I and Fe II lines in the region between 4300 and $4400 \AA$ indicates that WRAY 15-488 should have a spectral type earlier than G0. The strength of the G-band at $4300 \AA$ indicates that WRAY 15-488 has a spectral type earlier than F5. The G-band strength is consistent with spectral types F1-F3. The strength of the Fe II - Ti II and Y II - Fe II doublets at 4179 and $4173 \AA$, and the presence of the Sr II lines at 4078 and $4216 \AA$ rule out the luminosity classes $\mathrm{V}$ and $\mathrm{I}$ (they are too strong to be consistent with class V and too weak for being class I). The spectrum is best matched by a spectral template F2III. Szczerba et al. (2007) disqualified WRAY 15-488 as post-AGB star, indicating that is a T Tauri star based on the results of Gregorio-Hetem \& Hetem (2002). The latter authors suggest a spectral type F8, but do no specify the luminosity class. They report the observation of $\mathrm{H} \alpha$ in emission with an $E W$ of $-8 \AA$. The F8 spectral type is not consistent with the strength and shape of the G-band measured with our data. Gravity sensitive lines (e.g., Y II, Sr II) indicate that the star is not a dwarf. In summary, WRAY 15-488 is a giant star of spectral type F2IIIe. We note that in our spectra there is a marginal detection of the $\mathrm{Li}$ line at $6708 \AA$.

\section{WRAY 15-522}

WRAY 15-522 does not display $\mathrm{H} \alpha$ in emission. Its spectrum shows absorption features characteristic of stars with spectral types late $\mathrm{G}$ and early $\mathrm{K}$. The strength and width of the Ca I line at $4226 \AA$ indicate that WRAY 15-522 has a spectral type G8-K0. The strength of the Fe I line at $4271 \AA$ suggests that the spectral type is not later than $\mathrm{K} 0$. The strength and width of the Fe I lines at 4532, 4787, and $5079 \AA$, and the $\mathrm{Mg} \mathrm{I}$ line at $5711 \AA$ show that the spectral type is earlier than K0 but later than G8. The strength and width of Fe I+Ca I lines at $5270 \AA$, and the Fe I lines at 5329 and $5404 \AA$ indicate that the spectral type is earlier than K0. Therefore, we adopt the spectral type G9 for WRAY 15-522. The shape and width of the Sr II lines at 4078 and $4216 \AA$, the Y II line at $4375 \AA$, and the Ti II lines at 4400 and $4408 \AA$ (Ginestet et al. 1992, plate 44) indicate that the spectra is of a giant star. The strength of the Sr II+CN lines at $4216 \AA$ is too weak to be consistent with the luminosity class I and too strong to be consistent with the luminosity class V and IV. This indicates that WRAY 15-522 has a luminosity class III. We conclude that WRAY 15-522 has a spectral type G9III.

\section{WRAY 15-566}

WRAY 15-566 does not display $\mathrm{H} \alpha$ in emission. Since its spectrum displays VO and TiO molecular bands, WRAY 15-1566 should have a spectral type M5 or later. The spectrum does 
not display the Li line at $6708 \AA$ in absorption, thus showing that WRAY 15-566 is not a young star. The strength of the TiO bands at 4423, $4462 \AA$ indicates that WRAY 15-566 should have a spectral type M6 or earlier. The shape and strengths of the Mn I lines at 4031 and $4034 \AA$, the $\mathrm{Ca}$ I line at $4227 \AA$, the Fe I line at $4326 \AA$, and the TiO band at $4950 \AA$ show that WRAY 15-566 has a spectral type M6. The strength of the multiple absorption lines inside the molecular bands at 4400-6000 indicates that the source is a giant star of luminosity class III. We conclude that WRAY 15-566 has a spectral type M6III.

\section{WRAY 15-770}

WRAY 15-770 does not display $\mathrm{H} \alpha$ in emission. Its spectrum presents the $\mathrm{TiO}$ and $\mathrm{VO}$ molecular bands characteristic of stars of late-M spectral types. The narrow sodium lines at $5890 \AA$ indicate that WRAY 15-770 is a giant star of luminosity class III. The strength and shape of the CaI line at $4227 \AA$ indicate that WRAY 15-770 has a mid-M spectral type. Comparison of the $\mathrm{Ca}$ I line with spectral templates suggests a spectral type M6 or later but earlier than M8. The strength of the of the TiO bands indicates that the spectral type is later than M5. The absence of the sharp Ti I line at $4534 \AA$ indicates that WRAY 15-770 has a spectral type later than M6. The strength and shape of the molecular bands at $4740 \AA, 5740 \AA, 6000-6200 \AA$, and 7400-7500 are best matched by the spectral type M7III. We conclude that WRAY 15-770 has a spectral type M7III.

\section{WRAY 15-1104 = CD-55 $5174=C P D-555588$}

WRAY 15-1104 displays $\mathrm{H} \alpha$ in emission. He I absorption lines are present in the spectrum. The absence of the He II absorption lines, in particular the line at $4686 \AA$, indicates that the spectral type of WRAY 15-1104 is later than B0. The presence of He I absorption lines at 4009, 4121, and $4388 \AA$ shows that the spectral type is earlier than B5. The presence of N II line at $4631 \AA$ indicates that WRAY 15-1104 is a giant star of luminosity class I or II and rules out the spectral types B3 to B5. Given that the spectrum displays O II lines at 4317, 4320, 4246, $4349,4367,4639$, and $4642 \AA$, and that the Si IV line at $4089 \AA$ is present, WRAY 15-1104 should be of spectral type B1 and luminosity class I. The almost absent C II line at $4267 \AA$ and the strengths of the Si IV line at $4089 \AA$, the N II line at $4631 \AA$, and the O II lines at 4639 and $4642 \AA$ rule out the luminosity class Ib and indicate that WRAY 15-1104 should have a luminosity class Ia. Therefore, we conclude that WRAY 15-1104 has a spectral type B1Iae. Sarkar et al. (2005) studied WRAY 151104 and suggested a spectral type B1Ibe. As explained, our FEROS spectrum is better described by the luminosity class Ia than the luminosity class Ib. We note that the Sarkar et al. spectrum did not cover the wavelength range short-ward of $4900 \AA$, a region where important surface gravity indicators such as the Si IV line at $4089 \AA$, the N II line at $4631 \AA$, and the O II lines at 4639 and $4642 \AA$ are present.

\section{WRAY 15-1372 = CD-519596}

WRAY 15-1372 exhibits broad $\left(F W H M=382 \mathrm{~km} \mathrm{~s}^{-1}\right)$ double peaked $\mathrm{H} \alpha$ in emission. Its spectrum is flat and very few absorption lines are observed. Except for the He I lines at 4026 and $4471 \AA$ no other He I lines are present in the spectrum.
Therefore, WRAY 15-1372 should have a spectral type B6 or later. The strength of the $\mathrm{Mg}$ II line at $4481 \AA$ is similar to the strength of the He I line at $4471 \AA$. Thus, WRAY 15-1372 should have a spectral type earlier than B7. In consequence, WRAY 15-1372 has a spectral type B6. The absence of a strong C II line at $4267 \AA$ rules out the luminosity classes I and II. The similar width $\left(400 \mathrm{~km} \mathrm{~s}^{-1}\right)$ of the He I line at $4471 \AA$ and the Mg II line at $4481 \AA$ is not compatible with the luminosity class III. We conclude that WRAY 15-1372 has a spectral type B6Ve.

\section{WRAY 15-1435}

WRAY 15-1435 exhibits $\mathrm{H} \alpha, \mathrm{H} \beta, \mathrm{Ca}$ II at 8498 and $8662 \AA$ in emission. Its spectrum is rich in He I lines but no He II lines are observed. Since the He II line at $4686 \AA$ is absent, WRAY 15-14135 has a spectral type later than B0.5. The absence of the Mg II line at $4481 \AA$ shows that WRAY 15-14135 should have a spectral type earlier than B2 and rules out the luminosity classes I-III. These spectral characteristics indicate that WRAY 15-1435 has a spectral type B1Ve.

\section{WRAY 15-1650}

The spectrum of WRAY 15-1650 displays the TiO molecular bands characteristic of M-type stars. No $\mathrm{H} \alpha$ emission is observed and the Li line at $6708 \AA$ is absent. The strength of the molecular bands at 5000-8000 $\AA$ indicates that WRAY 15-1650 has a mid-M spectral type. The narrow Na I D lines observed at $5890 \AA$ indicate that WRAY $15-1650$ is a giant star of luminosity class III (see Montes et al. 1999). The shape of the spectrum and the strength of the molecular bands are best matched by the M6 spectra template: the bands at 4400 and $4500 \AA$ are stronger than in the M5III template and the bands at 6000-7000 $\AA$ are weaker than in the M7III template. We conclude that WRAY 15-1650 has a spectral type M6III.

\section{WRAY 15-1651}

WRAY 15-1651 displays $\mathrm{H} \alpha$ and $\mathrm{H} \beta$ emission lines. Paschen emission is observed starting at $8438 \AA$ up to $9000 \AA$. Na I D emission is observed at $5890 \AA$. The spectrum is flat and almost no absorption lines are observed. Since the $\mathrm{H} \alpha E W$ is of the order of $100 \AA$, it is likely that this object has a large accretion rate and that we are observing a strongly veiled spectrum. We performed the spectral classification based on the absence of strong spectral features that are observed in stars even with large accretion rates and strong veiling. No molecular bands are observed; thus WRAY 15-1651 should have a spectral type earlier than M. The Ca II line at $3933 \AA$ is not present and the Na I D lines have a FWHM $\left(48 \mathrm{~km} \mathrm{~s}^{-1}\right)$ lower than observed in $\mathrm{K}$ type stars. Therefore WRAY 15-1651 should have a spectral type earlier than K. There is no evidence for the strong metallic lines observed in F and G type stars; therefore WRAY 15-1561 should have a spectral type earlier than F. The lack of Ca II and Mg II line at $4481 \AA$ in absorption suggests that WRAY 15-1561 should have a spectral type earlier than A. Furthermore, the absence of the Mg II line at $4481 \AA$ indicates that WRAY 15-1561 should have an early B spectral type. The lack of the He II line at $4686 \AA$ suggests that WRAY 15-1561 is later than B0. We conclude that WRAY 15-1561 should have a spectral type B1 to B5. 
The lack of absorption lines does not allow us to constrain the luminosity class.

\section{WRAY 15-1702}

WRAY 15-1702 has the spectrum characteristic of mid M-type stars. It does not display $\mathrm{H} \alpha$ in emission and the Li line at $6708 \AA$ is absent. The narrow shape of the sodium lines at $5890 \AA$ indicates that WRAY 15-1702 is a giant star. The shape and relative intensity of the molecular bands at 4000, 5000, 6000, and $7000 \AA$ indicate that WRAY 15-1702 has a spectral type later than M5. The strength of the calcium lines at 8498 and $8662 \AA$ indicates that WRAY 15-1702 has a spectral type M6 or earlier. The spectrum that best match the strength of the molecular bands and the $100 \AA$ region around the $\mathrm{H} \beta, \mathrm{Na}$ I D, $\mathrm{H} \alpha$, and $\mathrm{Ca}$ II lines (see Montes et al. 1999) is the spectral type M6III. We conclude that WRAY 15-1702 has a spectral type M6III.

\section{Appendix 2. IR-emission and signs of star-formation activity in the vicinity of confirmed Herbig Ae/Be stars not associated with nearby SFRs}

We searched the Spitzer archive for $8.0 \mu \mathrm{m}$ imaging in the $20^{\prime}$ vicinity of the 11 confirmed Herbig Ae/Be stars with distances greater than $700 \mathrm{pc}$ (i.e. not associated to nearby SFRs). Additionally, we queried the SIMBAD data-base for astronomical objects in the $20^{\prime}$ vicinity suggesting star-formation activity such as HII regions, molecular clouds, dark clouds and young stellar objects. Our aim was first to check whether the sources are in empty regions or associated with large-scale IR emission and second to find evidence whether our sources might be members of distant SFRs. We used data from the Spitzer Galactic Legacy Infrared Mid-Plane Survey Extraordinaire (GLIMPSE) ${ }^{12}$ survey and, for querying the SIMBAD data base, the Aladin tool from the Centre des Données de Strasbourg (CDS $)^{13}$.

We found Spitzer $8 \mu \mathrm{m}$ images for 9 stars: HD 313571 , Hen 2-80, Hen 3-1121 N\&S, MWC 878, MWC 953, Th 17-35, WRAY 15-1372, and WRAY 15-1435. In Fig. 6, we show the $20^{\prime} \times 20^{\prime}$ vicinity of our sources. No data were available for Hen 3-823 and MWC 593. We find that 7 sources, Hen 2-80, Hen 3-1121 N\&S, HD 313571, MWC 953, WRAY 15-1435, and Th 17-35 are inside or close (separation $<5^{\prime}$ ) to regions with extended infrared (IR) emission and have in their vicinity astronomical sources characteristic of SFRs. We suggest that these 6 Herbig Ae/Be stars are be members of distant SFRs. However, we note that further study of the regions is required to validate this hypothesis. Two sources, MWC 878 and WRAY 15-1372, have no astronomical sources characteristic of SFRs and no or very weak nearby IR emission. They might be examples of "isolated" Herbig Ae/Be stars. In the following subsections, we discuss each source separately.

\section{HD 313571}

HD 313571 is embedded in a tenuous extended IR emission that increases of intensity towards the East. In the immediate vicinity of HD 313571 we observe at $20^{\prime \prime}$ and at $1,7^{\prime} \mathrm{SW}$ at PA $\sim 240^{\circ}$ two bright reflection nebulae. They are most-likely associated with the IRAS source 17580-2215. Palla et al. (1991) report a detection of $\mathrm{H}_{2} \mathrm{O}$ maser at $22 \mathrm{GHz}$ towards the position of

\footnotetext{
12 http://www.astro.wisc.edu/sirtf/

13 http://aladin.u-strasbg.fr
}

the nebulae. The dark nebulae LDN 237 is observed at $\sim 5 \mathrm{NW}$ $\left(\mathrm{PA} \sim 345^{\circ}\right)$ of HD 313571 . The presence of the reflexion nebulae and the detection of masers in the vicinity of HD 313571 indicate that the region is young and suggest that HD 313571 may be a member of this SFR.

\section{Hen 2-80}

Hen 2-80 is inside an IR emission nebulosity. This nebula has several components: the reflection nebulae $\mathrm{VdBH} 57 \mathrm{~b}$ and BRAN 388, and at $2^{\prime} \mathrm{SW}$ and $\mathrm{PA} \sim 115^{\circ}$ the reflection nebulae $\mathrm{VdBH} 57 \mathrm{~d}$. The presence of reflection nebulae provides further evidence of the young nature of Hen 2-80. Given the diversity of reflection nebulae observed and the presence of several IRSpitzer sources in the region, we speculate that Hen 2-80 is a region where a stellar cluster is in formation. Further mid-IR and sub-mm imaging would be advisable to determine the number of young sources present in the region.

\section{Hen 3-1121 N and Hen 3-1121 S}

Hen 3-1121 N\&S are located at the N-E of a large $\left(20^{\prime} \times 20^{\prime}\right)$ ring-shaped IR emission region. A SIMBAD database search shows that this region embeds several molecular clouds (G328.3-0.5-46.0, G328.2-0.5-38.0, G328.2-0.5-40.3.0, G328.2-0.5-43.3, G328.2-0.5-45.8, G328.2-0.5-46.1); OH and $\mathrm{CH} 3 \mathrm{OH}$ masers (Caswell OH 328.237-00.547, OH 328.25-00.5, $\mathrm{CH} 3 \mathrm{OH} 328.25-00.53, \mathrm{CH} 3 \mathrm{OH} 328.24-00.55, \mathrm{CH} 3 \mathrm{OH} 328.23-$ $00.53)$; the reflection nebula GN 15.53.6; and the HII regions GAL 328.2-00.5, IRAS 15541-5349 (at the center), and IRAS 15539-5353. This ensemble of objects indicates unmistakably that this region is a region of active star formation. Since the spectroscopy parallax distances of Hen 3-1121 S\&N $\left(3.1_{-0.8}^{+1.0}\right.$ and $3.2_{-2.1}^{+6.3} \mathrm{kpc}$ respectively) are consistent with the kinematic distance of $3.0 \mathrm{kpc}$ of the G328.236-0.547 maser (Phillips et al. 1998), we suggest that Hen 3-1121 N\&S are members of this SFR.

\section{MWC 878}

West from MWC 878 we find a tenuous large scale infrared emission. MWC 878 is located just at the outskirts of this IRemission. A search in the SIMBAD database shows no sources characteristic of SFRs in the $20^{\prime}$ vicinity. Therefore, we suggest that MWC 878 might be an example of an "isolated" Herbig Ae/Be star.

\section{MWC 953}

MWC 953 is embedded in an extended IR emission with a filamentary geometry. At $\sim 1^{\prime} \mathrm{N}$ and $6^{\prime} \mathrm{S}$ of MWC 953 we find regions strongly resembling shocks. At $5^{\prime} \mathrm{NW}$ and $\mathrm{PA} \sim 290^{\circ}$ an extended dark nebula (G028.67+00.13) of size $\sim 8^{\prime} \times 3.5^{\prime}$ is observed. In the region several YSOs are present (e.g., ISOGAL-P J184333.9-034459, ISOGAL-P J184329.0-034522). The region is rich in $\mathrm{X}$-ray sources. The molecular cloud SRBY 152 is observed 5.5' SW of MWC $953\left(\mathrm{PA} \sim 216^{\circ}\right)$. The presence of these objects indicates that the region is active in star formation. We suggest that MWC 953 might be associated to the region. Further studies of the stellar content are required to validate this conclusion. 

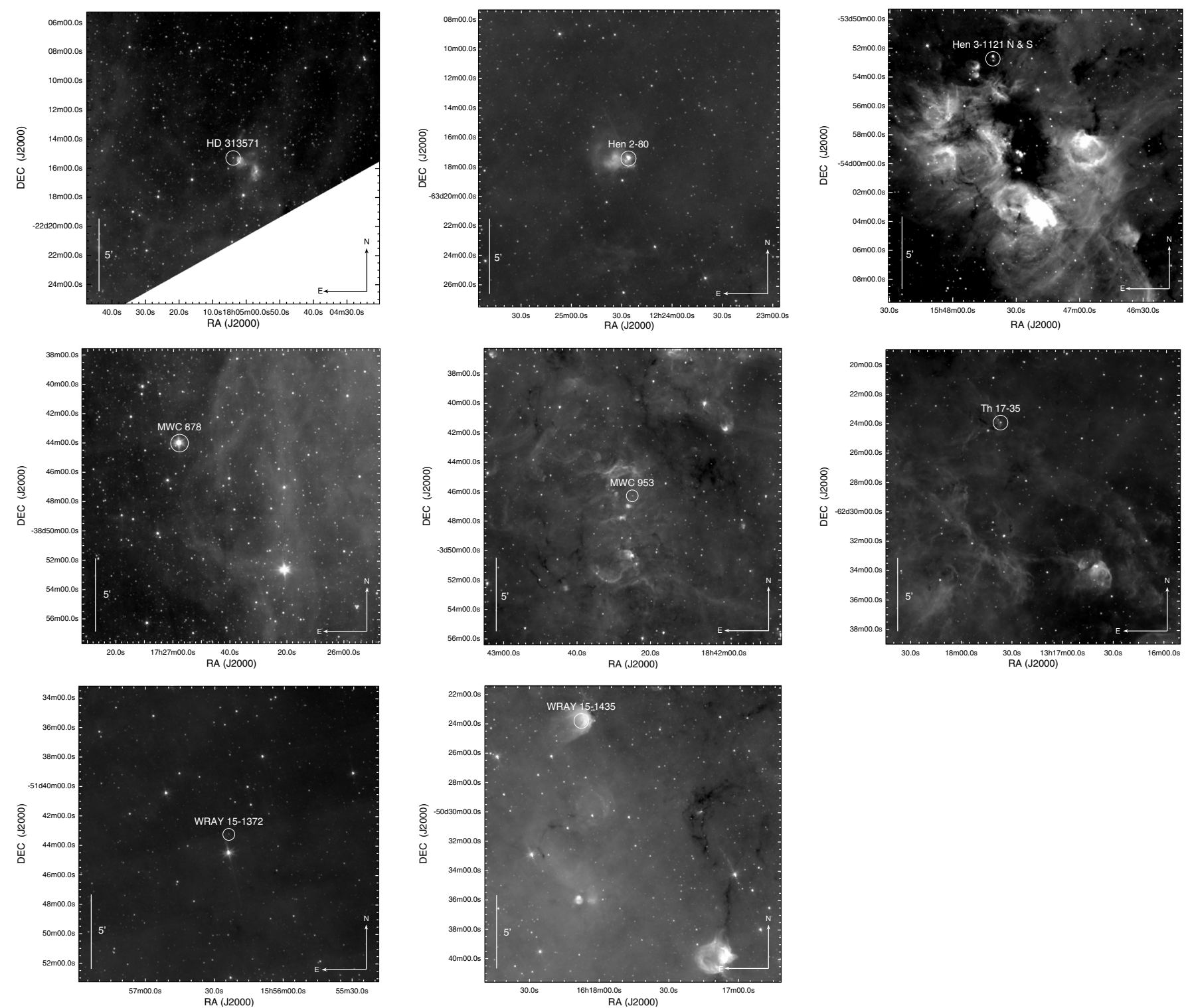

Fig. 6. Spitzer $8.0 \mu \mathrm{m}$ images of the $20^{\prime} \times 20^{\prime}$ vicinity of the confirmed Herbig Ae/Be stars not associated with nearby SFRs.

\section{Th 17-35}

The Spitzer image of the $20^{\prime}$ vicinity of Th $17-35$ reveals a large-scale filamentary IR-emission. The SIMBAD database shows the presence of the cloud (of unknown nature) SFO 73 at position of Th 17-35, the molecular clouds G306.2+0.2-42.0, G306.2+0.2-29.2, and G306.2+0.2-28.6 ( $\sim 6^{\prime}$ south $)$, the dark nebula DCld $306.3+00.2\left(10^{\prime} \mathrm{SE}, \mathrm{PA} \sim 215^{\circ}\right)$, and a region at $12^{\prime} \mathrm{SW}\left(\mathrm{PA} \sim 215^{\circ}\right)$ comprehending the reflection nebula ESO 132-4, the cloud (of unknown nature) SFO 72, the dark cloud DCld 306.2+00.1 and the emission line star Th 17-33. This ensemble of characteristics indicates that this is an active region of star-formation. Since Th 17-35 is a young star it is likely that it is a member of this region.

\section{WRAY 15-1372}

WRAY 15-1372 is inside a very tenuous IR-emission. The SIMBAD database does not display astronomical objects associated with SFRs in the 20' vicinity of WRAY 15-1372. These characteristics suggest that WRAY 15-1372 may be an "isolated" Herbig Ae/Be star.

\section{WRAY 15-1435}

WRAY $15-1435$ is positionally coincident with a reflection nebula (CSI-50-16095). All the region is embedded in a tenuous large scale IR emission. We observe in the region the molecular cloud G332.3+0.5-96.2 approximately 6' south of WRAY 15-1435, and the the high-mass protostellar candidate IRAS 16082-5031 (Fontani et al. 2005) 20' SW. At 10' SW and at $\mathrm{PA} \sim 235^{\circ}$ a dark absorption region with a shape resembling a sickle is observed. We suggest that WRAY 15-1435 is a member of this star-formation region. However, further study is required to determine whether the objects observed in the region are physically related. 